\title{
PETROV-GALERKIN AND SPECTRAL COLLOCATION METHODS FOR DISTRIBUTED ORDER DIFFERENTIAL EQUATIONS
}

\author{
EHSAN KHARAZMI *, MOHSEN ZAYERNOURI ${ }^{\dagger}$ AND GEORGE EM KARNIADAKIS *
}

\begin{abstract}
Distributed order fractional operators offer a rigorous tool for mathematical modelling of multiphysics phenomena, where the differential orders are distributed over a range of values rather than being just a fixed integer/fraction as it is in standard/fractional ODEs/PDEs. We develop two spectrally-accurate schemes, namely a Petrov-Galerkin spectral method and a spectral collocation method for distributed order fractional differential equations. These schemes are developed based on the fractional Sturm-Liouville eigen-problems (FSLPs) [61]. In the Petrov-Galerkin method, we employ fractional (non-polynomial) basis functions, called Jacobi poly-fractonomials, which are the eigenfunctions of the FSLP of first kind, while, we employ another space of test functions as the span of poly-fractonomial eigenfunctions of the FSLP of second kind. We define the underlying distributed Sobolev space and the associated norms, where we carry out the corresponding discrete stability and error analyses of the proposed scheme. In the collocation scheme, we employ fractional (non-polynomial) Lagrange interpolants satisfying the Kronecker delta property at the collocation points. Subsequently, we obtain the corresponding distributed differentiation matrices to be employed in the discretization of the strong problem. We perform systematic numerical tests to demonstrate the efficiency and conditioning of each method.
\end{abstract}

Key words. Distributed Sobolev space, distributed bilinear forms, modal/nodal basis, fractional Lagrange interpolants, spectral convergence, stability/error analysis, uncertainty quantification

AMS subject classifications. 34L10, 58C40, 34K28, 65M70, 65M60

1. Introduction. Fractional differential equations (FDEs) seamlessly generalize the notion of standard (integer-order) differential equations to those of fractional order [48, 32, 46]. There exists plenty experimental evidence revealing the anomalous transport and nonlocal history dependent effect in complex physical systems. Fractional calculus and FDEs open up new possibilities for robust modeling of such complex multi-scale problems. Examples include: non-Gaussian (Lévy flights) processes in turbulent flows [50, 29, 9], non-Newtonian fluids and rheology [27, 45], non-Brownian transport phenomena in porous and disordered materials [5, 41], and non-Markovian processes in multi-scale complex fluids and multi-phase applications [28]. Over the past two decades, an extensive amount of work has been done developing numerical schemes for FDEs such as variational iteration method [25], homotopy perturbation method [54], Adomians decomposition method [26], homotopy analysis method [22] and collocation method [47]. While most of the attention has been devoted to the finite difference methods (FDMs), [38, 39, 49, 52, 42, 21, 13, 33, 53, 37, 55, 57, 24, 7, 67, 66], recent works have focused on exploring the potential efficiency of spectral methods and their inherent global nature as more suitable to discretizing FDEs, see e.g., [52, 47, 37, 30, 31, 35, 36, 8, 56, 6].

Two new spectral theories on fractional and tempered fractional Sturm-Liouville problems (TFSLPs) have been recently developed by Zayernouri et al. in [61, 59]. This approach first fractionalizes and then tempers the well-known theory of Sturm-Liouville eigenproblems. The explicit eigenfunctions of TFSLPs are analytically obtained in terms of tempered Jacobi poly-fractonomials. Recently, in [63, 60, 62], Jacobi poly-fractonomials were successfully employed in developing a series of high-order and efficient Petrov-Galerkin

\footnotetext{
${ }^{*}$ Department of Computational Mathematics, Science, and, Engineering \& Department of Mechanical Engineering, Michigan State University, 428 S Shaw Lane, East Lansing, MI 48824, USA

$\dagger$ Department of Computational Mathematics, Science, and, Engineering \& Department of Mechanical Engineering, Michigan State University, 428 S Shaw Lane, East Lansing, MI 48824, USA, Corresponding author; zayern@msu.edu

${ }^{\ddagger}$ Division of Applied Mathematics, Brown University, 182 George Street, Providence, RI 02912, USA
} 
spectral and discontinuous spectral element methods of Galerkin and Petrov-Galerkin projection type for fractional ODEs. To treat nonlinear problems the collocation schemes are relatively easy to implement. Khader in [30] presented a Chebyshev collocation method for the discretization of the space-fractional diffusion equation. More recently, Khader and Hendy [31] developed a Legendre pseudospectral method for fractional-order delay differential equations. For fast treatment of nonlinear and multi-term fractional PDEs such as the fractional Burgers equation, a new spectral method, called fractional spectral collocation method, was developed in [64]. This new class of collocation schemes introduces a new family of fractional Lagrange interpolants, mimicking the structure of the Jacobi polyfractonomials. For variable-order fractional PDEs, a fast and spectrally accurate collocation method was developed and implemented in [65].

Distributed order fractional operators offer a rigorous tool for mathematical modeling of multi-physics phenomena. In this case, the differential order is distributed over a range of values rather than being just a fixed fraction as it is in standard/fractional ODEs/PDEs. There is a rapidly growing interest in the use of fractional derivatives in the construction of mathematical models, which contain distributed order terms of the form

$$
\int_{\sigma_{1}}^{\sigma_{2}} \phi(\sigma){ }_{a}^{*} \mathcal{D}_{t}^{\sigma} u(t) d \sigma=f(t), \quad t>a,
$$

in the field of uncertainty quantification as the inherent uncertainty of experimental data can be directly incorporated into the differential operators; see [40, 11, 3, 4, 51], for some work on numerical methods. Almost all of the numerical schemes developed for such models are finite-difference methods. While the treatment of fractional differential equations with a fixed fractional order could be memory demanding due to the locality of these methods and their low-accuracy, the main challenge remains the additional effect of the discretization of the distributed order model, which may lead to exceeding computational cost of numerical simulations.

To the best of our knowledge, the first numerical study of distributed order differential equations (DODEs) was performed by Diethelm and Ford in [10], where a two-stage basic framework was developed. In the first stage, the distributed order differentiation term was approximated using a quadrature rule, and in the second stage, a suitable multi-term numerical method was employed. They later performed the corresponding error analysis of the method in [12]. Subsequently, most of the numerical studies have followed the same approach yet they vary in the discretization method in the second stage. The distributed order timefractional diffusion equation was numerically studied in [16] and the corresponding stability and convergence study of the scheme was provided in [17]. Adding a nonlinear source, [44] studied the distributed order reaction diffusion equation following the same scheme. In [34], the second stage of the distributed order diffusion equation was established using a reproducing kernel method. The distributed order time fractional diffusion-wave equation was investigated by developing a compact difference scheme in [58]. Other numerical studies include: an implicit numerical method of a temporal distributed order and two-sided space-fractional advection-dispersion equation in [23], high-order difference schemes in [18], alternating direction implicit (ADI) difference schemes with the extrapolation method for one-dimensional case in [20] and two-dimensional problem in [19], and an operational matrix technique in [14].

In this paper, we first introduce the distributed Sobolev spaces and their associated norms. We show their equivalence to the defined left-side and right-side norms as well. By employing Riemann-Liouville derivatives, we define the distributed order differential equation and then obtain its variational form. We develop a Petrov-Galerkin (PG) spectral method following the recent theory of fractional Sturm-Liouville eigen-problems (FSLP) in [61] and 
employ the corresponding eigenfunctions, namely the Jacobi Poly-fractonomials of first kind as the bases and the Jacobi Poly-fractonomials of second kind as test functions. We develop a spectrally accurate Gauss-Legendre quadrature rule in the construction of the linear system, where we investigate the stability and error analysis of the scheme. In addition, we construct a spectrally-accurate fractional spectral collocation scheme, where we employ fractional Lagrange interpolants satisfying the Kronecker delta property at the collocation points, and then, we obtain the corresponding fractional differentiation matrices. We demonstrate the computational efficiency of both schemes considering several numerical examples and distribution functions.

The organization of the paper is as follows: section 2 provides preliminary definitions along with useful lemmas. We recall fractional Sobolev spaces, and then, introduce their generalization to so called distributed Sobolev space and associated norms, which provides the natural setting of our problem in this study. We furthermore obtain some equivalent norms to facilitate the corresponding analysis of our methods. In section 3 , we derive and discretize the corresponding variational form of the problem and subsequently we prove the stability and convergence rate of the scheme. In addition, we develop a fractional collocation method in section 4 and test the performance of the two methods in section 5 . We conclude the paper with a summary and conclusion.

2. Definitions. Let $\xi \in[-1,1]$. Then, the left-sided and right-sided Riemann-Liouville integral of order $\sigma, n-1<\sigma \leq n, n \in \mathbb{N}$, are defined (see e.g., [43, 46]) respectively as

$$
\begin{gathered}
\left({ }_{-1}^{R L} \mathcal{I}_{\xi}^{\sigma}\right) u(\xi)=\frac{1}{\Gamma(\sigma)} \int_{-1}^{\xi} \frac{u(s) d s}{(\xi-s)^{n-\sigma}}, \quad \xi>-1, \\
\left({ }_{\xi}^{R L} \mathcal{I}_{1}^{\sigma}\right) u(\xi)=\frac{1}{\Gamma(\sigma)} \int_{\xi}^{1} \frac{u(s) d s}{(s-\xi)^{n-\sigma}}, \quad \xi<1 .
\end{gathered}
$$

The corresponding left-sided and right-sided fractional derivative of order $\sigma$ are then defined, as

$$
\begin{gathered}
\left({ }_{-1}^{R L} \mathcal{D}_{\xi}^{\sigma}\right) u(\xi)=\frac{d^{n}}{d \xi^{n}}\left({ }_{-1}^{R L} \mathcal{I}_{\xi}^{n-\sigma} u\right)(\xi)=\frac{1}{\Gamma(n-\sigma)} \frac{d^{n}}{d \xi^{n}} \int_{-1}^{\xi} \frac{u(s) d s}{(\xi-s)^{\sigma+1-n}}, \quad \xi>-1, \\
\left({ }_{\xi}^{R L} \mathcal{D}_{1}^{\sigma}\right) u(\xi)=\frac{(-d)^{n}}{d \xi^{n}}\left({ }_{\xi}^{R L} \mathcal{I}_{1}^{n-\sigma} u\right)(\xi)=\frac{1}{\Gamma(n-\sigma)} \frac{(-d)^{n}}{d \xi^{n}} \int_{\xi}^{1} \frac{u(s) d s}{(s-\xi)^{\sigma+1-n}}, \quad \xi<1,
\end{gathered}
$$

respectively. We recall a useful property of the Riemann-Liouville fractional derivatives [46]. Assume that $0<p<1$ and $0<q<1$ and $g\left(x_{L}\right)=0 x>x_{L}$, then

$$
{ }_{x_{L}} \mathcal{D}_{x}^{p+q} g(x)=\left({ }_{x_{L}} \mathcal{D}_{x}^{p}{ }_{x_{L}} \mathcal{D}_{x}^{q} g\right)(x)=\left({ }_{x_{L}} \mathcal{D}_{x}^{q} x_{x_{L}} \mathcal{D}_{x}^{p} g\right)(x) .
$$

An alternative approach in defining the fractional derivatives is to begin with the leftsided Caputo derivatives of order $\sigma, n-1<\sigma \leq n, n \in \mathbb{N}$, defined, as

$$
\left({ }_{-1}^{C} \mathcal{D}_{\xi}^{\sigma} u\right)(\xi)=\left({ }_{-1} I_{\xi}^{n-\sigma} \frac{d^{n} u}{d \xi^{n}}\right)(\xi)=\frac{1}{\Gamma(n-\sigma)} \int_{-1}^{\xi} \frac{u^{(n)}(s) d s}{(\xi-s)^{\sigma+1-n}}, \quad \xi>-1 .
$$

By performing an affine mapping from the standard domain $[-1,1]$ to the interval $t \in[a, b]$, we obtain

$$
\begin{aligned}
{ }_{a}^{R L} \mathcal{D}_{t}^{\sigma} u & =\left(\frac{2}{b-a}\right)^{\sigma}\left({ }_{-1}^{R L} \mathcal{D}_{\xi}^{\sigma} u\right)(\xi), \\
{ }_{a}^{C} \mathcal{D}_{t}^{\sigma} u & =\left(\frac{2}{b-a}\right){ }^{\sigma}\left({ }_{-1}^{C} \mathcal{D}_{\xi}^{\sigma} u\right)(\xi) .
\end{aligned}
$$


Hence, we can perform the operations in the standard domain only once for any given $\sigma$ and efficiently utilize them on any arbitrary interval without resorting to repeating the calculations. Moreover, the corresponding relationship between the Riemann-Liouville and Caputo fractional derivatives in $[a, b]$ for any $\sigma \in(0,1)$ is given by

$$
\left({ }_{a}^{R L} \mathcal{D}_{t}^{\sigma} u\right)(t)=\frac{u(a)}{\Gamma(1-\sigma)(t-a)^{\sigma}}+\left({ }_{a}^{C} \mathcal{D}_{t}^{\sigma} u\right)(t)
$$

LEMMA 2.1. Let $\sigma, \mu>0$. The fractional derivative of the Jacobi poly-fractonomials, [61], of first $(i=1)$ and second kind $(i=2)$ are given by

$$
{ }^{R L} \mathcal{D}^{\sigma}\left\{{ }^{(i)} \mathcal{P}_{n}^{\mu}(\xi)\right\}=\frac{\Gamma(n+\mu)}{\Gamma(n+\mu-\sigma)}{ }^{(i)} \mathcal{P}_{n}^{\mu-\sigma}(\xi),
$$

and are also of Jacobi poly-fractonomial type, where ${ }^{R L} \mathcal{D}^{\sigma} \equiv{ }_{-1}^{R L} \mathcal{D}_{x}^{\sigma}$ when $i=1,{ }^{R L} \mathcal{D}^{\sigma} \equiv$ ${ }_{x}^{R L} \mathcal{D}_{1}^{\sigma}$ when $i=2$.

Proof. See Appendix (A).

REMARK 2.2. Lemma 2.1 shows that the structure of Jacobi poly-fractonomials is preserved under the action of fractional derivatives. Moreover, we note that when $\sigma=\mu$ in Lemma 2.1. the fractional derivatives of Jacobi poly-fractonomials are obtained in terms of Legendre polynomials, which has been reported in [61].

2.1. Fractional Sobolev Spaces. By $H^{s}(\mathbb{R}), s \geq 0$, we denote the fractional Sobolev space on $\mathbb{R}$, defined as

$$
H^{s}(\mathbb{R})=\left\{v \in L^{2}(\mathbb{R}) \mid\left(1+|\omega|^{2}\right)^{\frac{s}{2}} \mathcal{F}(v)(\omega) \in L^{2}(\mathbb{R})\right\},
$$

which is endowed with the norm

$$
\|\cdot\|_{s, \mathbb{R}}=\left\|\left(1+|\omega|^{2}\right)^{\frac{s}{2}} \mathcal{F}(\cdot)(\omega)\right\|_{L^{2}(\mathbb{R})},
$$

where $\mathcal{F}(v)$ represents the Fourier transform of $v$. Subsequently, we denote by $H^{s}(I), s \geq 0$ the fractional Sobolev space on any finite closed interval $I$, defined as

$$
H^{s}(I)=\left\{v \in L^{2}(\mathbb{R}) \mid \exists \tilde{v} \in H^{s}(\mathbb{R}) \text { s.t. }\left.\tilde{v}\right|_{I}=v\right\},
$$

with the norm

$$
\|\cdot\|_{s, I}=\inf _{\tilde{v} \in H^{s}(\mathbb{R}),\left.\tilde{v}\right|_{I}=(\cdot)}\|\tilde{v}\|_{s, \mathbb{R}} .
$$

We note that the definition of $H^{s}(I)$ and the corresponding norm relies on the Fourier transformation of the function. Other useful norms associated with $H^{s}(I)$, e.g., when $I=\left[x_{L}, x_{R}\right]$, have been also introduced in [35],

$$
\begin{gathered}
\|\cdot\|_{l, s, I}=\left(\|\cdot\|_{L^{2}(I)}^{2}+\left\|{ }_{x_{L}}^{R L} \mathcal{D}_{x}^{\mu}(\cdot)\right\|_{L^{2}(I)}^{2}\right)^{\frac{1}{2}}, \\
\|\cdot\|_{r, s, I}=\left(\|\cdot\|_{L^{2}(I)}^{2}+\left\|{ }_{x}^{R L} \mathcal{D}_{x_{R}}^{\mu}(\cdot)\right\|_{L^{2}(I)}^{2}\right)^{\frac{1}{2}}
\end{gathered}
$$

such that the left-side $\|\cdot\|_{l, s, I}$, the right-sided $\|\cdot\|_{r, s, I}$, and $\|\cdot\|_{s, I}$ are shown to be equivalent.

Next, let $\phi \in L^{1}\left(\left[\alpha_{\text {min }}, \alpha_{\text {max }}\right]\right), 0 \leq \alpha_{\text {min }}<\alpha_{\text {max }}$, be nonnegative. By ${ }^{\phi} \mathcal{H}(\mathbb{R})$, we denote the distributed fractional Sobolev space on $\mathbb{R}$, defined as

$$
{ }^{\phi} \mathcal{H}(\mathbb{R})=\left\{v \in L^{2}(\mathbb{R}) \mid \int_{\alpha_{\min }}^{\alpha_{\max }}\left[\phi(\alpha)\left(1+|\omega|^{2}\right)^{\alpha}\right]^{\frac{1}{2}} \mathcal{F}(v)(\omega) d \alpha \in L^{2}(\mathbb{R})\right\}
$$



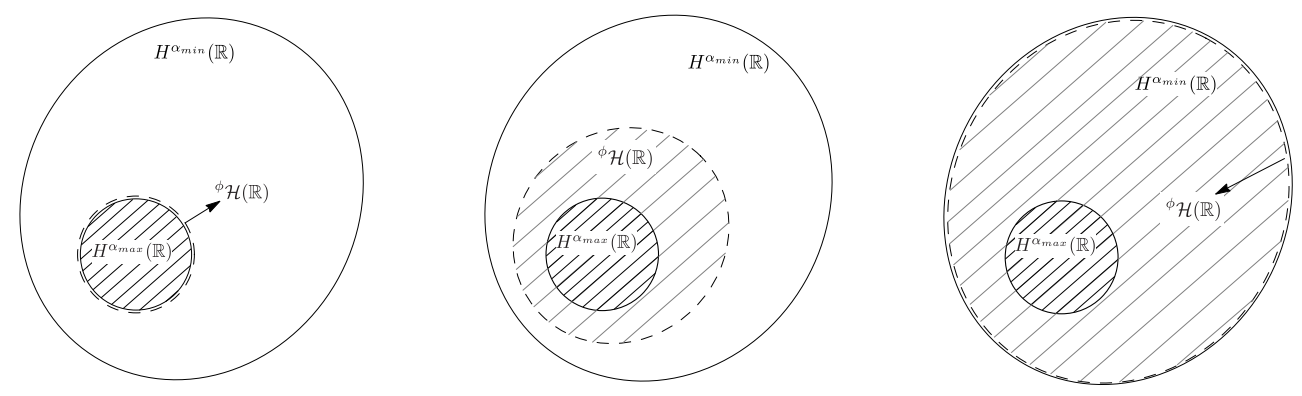

Fig. 2.1: Schematic of distributed fractional Sobolev space ${ }^{\phi} \mathcal{H}(\mathbb{R})$ : (left) $\phi=\delta\left(\alpha-\alpha_{\max }\right.$ ) hence ${ }^{\phi} \mathcal{H}(\mathbb{R})=H^{\alpha_{\max }}(\mathbb{R}) ;\left(\right.$ middle) $\phi$ defined on a compact support in $\left[\alpha_{\min }, \alpha_{\max }\right]$, hence, ${ }^{\phi} \mathcal{H}(\mathbb{R}) \supset H^{\alpha_{\max }}(\mathbb{R}) ;$ (right) $\phi=\delta\left(\alpha-\alpha_{\text {min }}\right)$, where ${ }^{\phi} \mathcal{H}(\mathbb{R})=H^{\alpha_{\text {min }}}(\mathbb{R})$.

which is endowed with the norm

$$
\|\cdot\|_{\phi, \mathbb{R}}=\left(\int_{\alpha_{\min }}^{\alpha_{\max }} \phi(\alpha)\left\|\left(1+|\omega|^{2}\right)^{\frac{\alpha}{2}} \mathcal{F}(\cdot)(\omega)\right\|_{L^{2}(\mathbb{R})}^{2} d \alpha\right)^{\frac{1}{2}} .
$$

Subsequently, we denote by ${ }^{\phi} \mathcal{H}(I)$ the distributed fractional Sobolev space on the finite closed interval $I$, defined as

$$
{ }^{\phi} \mathcal{H}(I)=\left\{v \in L^{2}(\mathbb{R}) \mid \exists \tilde{v} \in{ }^{\phi} \mathcal{H}(\mathbb{R}) \text { s.t. }\left.\tilde{v}\right|_{I}=v\right\},
$$

with the norm

$$
\|\cdot\|_{\phi, I}=\inf _{\tilde{v} \epsilon^{\mathcal{H}_{\mathcal{H}}(\mathbb{R}), \tilde{v} l}=(\cdot)}\|\tilde{v}\|_{\phi, \mathbb{R}}
$$

Moreover, we introduce the following useful norms, associated with ${ }^{\phi} \mathcal{H}(I)$ :

$$
\|\cdot\|_{l, \phi, I}=\left(\|\cdot\|_{L^{2}(I)}^{2}+\int_{\alpha_{\min }}^{\alpha_{\max }} \phi(\alpha)\left\|_{x_{L}}^{R L} \mathcal{D}_{x}^{\alpha}(\cdot)\right\|_{L^{2}(I)}^{2} d \alpha\right)^{\frac{1}{2}},
$$

and

$$
\|\cdot\|_{r, \phi, I}=\left(\|\cdot\|_{L^{2}(I)}^{2}+\int_{\alpha_{\min }}^{\alpha_{\max }} \phi(\alpha)\left\|_{x_{L}}^{R L} \mathcal{D}_{x}^{\alpha}(\cdot)\right\|_{L^{2}(I)}^{2} d \alpha\right)^{\frac{1}{2}} .
$$

We note that when $\phi>0$ is continuous in $I,{ }^{\phi} \mathcal{H}(\mathbb{R})$ is equivalent to $H^{\alpha_{\max }}(\mathbb{R})$. However, in general, the choice of $\phi$ can arbitrarily confine the domain of integration in practice. In other words, $\alpha_{\min }$ and $\alpha_{\max }$ are only the theoretical lower and upper terminals in the definition of distributed order fractional derivative. For instance, in a distributed sub-diffusion problem, the temporal derivative is associated with $\alpha_{\min }=0$ and $\alpha_{\max }=1$, and in a super-diffusion problem, the theoretical upper terminal $\alpha_{\max }=2$. In this study we particularly aim to let $\phi$ be defined in any possible subset of the interval $\left[\alpha_{\min }, \alpha_{\max }\right]$. Hence, in each realization of a physical process (e.g. sub- or super-diffusion) $\phi$ can be obtained from data, where the theoretical setting of the problem remains invariant yet requiring the solution to have less regularity $\left(\right.$ since $^{\phi} \mathcal{H}(\mathbb{R}) \supset H^{\alpha_{\max }}(\mathbb{R})$ in general, see Fig 2.1 . 
In the following theorem, we prove the equivalence (shown by the notation $\sim$ ) of the aforementioned norms.

Theorem 2.1. Let $\phi \in L^{1}\left(\left[\alpha_{\min }, \alpha_{\max }\right]\right)$ be non-negative. Then, the norms $\|\cdot\|_{\phi, I},\|\cdot\|_{l, \phi, I}$, and $\|\cdot\|_{r, \phi, I}$ are equivalent.

Proof. See Appendix (B).

LEMmA 2.3. [35]: For all $0<\alpha \leq 1$, if $u \in H^{1}([a, b])$ such that $u(a)=0$, and $w \in$ $H^{\alpha / 2}([a, b])$, then

$$
\left({ }_{a} \mathcal{D}_{s}^{\alpha} u, w\right)_{\Omega}=\left({ }_{a} \mathcal{D}_{s}^{\alpha / 2} u,{ }_{s} \mathcal{D}_{b}^{\alpha / 2} w\right)_{\Omega},
$$

where $(\cdot, \cdot)_{\Omega}$ represents the standard inner product in $\Omega=[a, b]$.

Lemma 2.4. Let $1 / 2<\mu<1, a$ and $b$ be arbitrary finite or infinite real numbers. Assume $u \in H^{2 \mu}(a, b)$ such that $u(a)=0$, also ${ }_{x} \mathcal{D}_{b}^{\mu} v$ is integrable in $(a, b)$ such that $v(b)=0$. Then

$$
\left({ }_{a} \mathcal{D}_{x}^{2 \mu} u, v\right)=\left({ }_{a} \mathcal{D}_{x}^{\mu} u,{ }_{x} \mathcal{D}_{b}^{\mu} v\right)
$$

Proof. See Appendix (C). $\square$

REMARK 2.5. Unlike other existing proofs (e.g., see Proposition 1 in [68]), our proof requires $v(x)$ to only vanish at the right boundary (note that $v(a)$ can be non-zero), moreover, we only require the $\mu$-th derivative (rather than the first derivative) of $v(x)$ to be integrable in $(a, b)$.

2.2. Problem Definition. Following [2], let $\alpha \mapsto \phi(\alpha)$ be a continuous mapping in $\left[\alpha_{\min }, \alpha_{\max }\right]$. Then, we define the distributed order fractional derivative as

$$
D \mathcal{D}_{\phi} u(t)=\int_{\alpha_{\min }}^{\alpha_{\max }} \phi(\alpha){ }_{a}^{*} \mathcal{D}_{t}^{\alpha} u(t) d \alpha, \quad t>a,
$$

where ${ }_{a}^{*} \mathcal{D}_{t}^{\alpha}$ denotes Riemann-Liouville fractional derivative of order $\alpha$. Next, we aim to solve the following differential equation of distributed order:

$$
\begin{aligned}
{ }^{D} \mathcal{D}_{\phi} u(t) & =f(t ; u), \quad \forall t \in(0, T], \\
u(0) & =0, \quad\left(\alpha_{\text {max }}\right) \in(0,1], \\
u(0)=\left.\frac{d u}{d t}\right|_{t=0} & =0, \quad\left(\alpha_{\text {max }}\right) \in(1,2] .
\end{aligned}
$$

In the sequel, we present different approaches to discretize the aforementioned differential operator. Due to 2.9], the Caputo and Riemann-Liouville fractional derivatives of order $\alpha \in(0,1)$ coincide with each other when $u(a)=0$. Therefore, in this study, we employ the definition of the distributed fractional derivatives of Riemann-Liouville sense and remove the pre-superscript $R L$ for simplicity.

3. Variational Formulation. In order to obtain the variational form we multiply 2.26) by a proper test function $v$ (defined later) and integrate over the computational domain:

$$
\int_{\Omega}{ }^{D} \mathcal{D}_{\phi} u(t) v(t) d \Omega=\int_{\Omega} f(t ; u) v(t) d \Omega .
$$

Using the definition of distributed order fractional derivatives defined in 2.25 we get 


$$
\int_{\alpha_{\min }}^{\alpha_{\max }} \phi(\alpha) \int_{\Omega} 0 \mathcal{D}_{t}^{\alpha} u(t) v(t) d \Omega d \alpha=\int_{\Omega} f(t ; u) v(t) d \Omega
$$

where

$$
\forall \alpha \in\left(\alpha_{\min }, \alpha_{\max }\right), \quad \int_{\Omega}{ }_{0} \mathcal{D}_{t}^{\alpha} u v d \Omega=\left({ }_{0} \mathcal{D}_{t}^{\alpha} u, v\right)_{\Omega}
$$

denotes the well-known $L^{2}$-inner product. Given the initial conditions 2.27) and/or 2.28) and by Lemmas (2.3), we define the bilinear form associated with $\alpha \in\left(\alpha_{\min }, \alpha_{\max }\right)$ as

$$
a^{(\alpha)}(u, v)=\left({ }_{0} \mathcal{D}_{t}^{\alpha} u, v\right)_{\Omega}=\left({ }_{0} \mathcal{D}_{t}^{\alpha / 2} u,{ }_{t} \mathcal{D}_{T}^{\alpha / 2} v\right)_{\Omega} .
$$

We choose $v$ such that $v(T)=0$ and ${ }_{t} \mathcal{D}_{T}^{\alpha / 2} v$ is integrable in $\Omega \forall \alpha \in\left(\alpha_{\min }, \alpha_{\max }\right)$. Moreover, let $U$ be the solution space, defined as

$$
\begin{aligned}
& U=\left\{u \in L^{2}(\Omega): \quad \sqrt{\int_{\alpha_{\min }}^{\alpha_{\max }} \phi(\alpha)\left\|_{0} \mathcal{D}_{t}^{\alpha / 2} u\right\|_{L^{2}(\Omega)}^{2} d \alpha}<\infty\right. \\
& \left.\begin{array}{lll}
\text { s.t. } & u(0)=0 & \text { if } \alpha_{\max } \in(0,1] \\
& u(0)=\left.\frac{d u}{d t}\right|_{t=0}=0 & \text { if } \alpha_{\max } \in(1,2]
\end{array}\right\}
\end{aligned}
$$

and let $V$ be the test function space given by

$$
V=\left\{v \in L^{2}(\Omega): \sqrt{\int_{\alpha_{\min }}^{\alpha_{\max }} \phi(\alpha)\left\|_{t^{\prime}} \mathcal{D}_{T}^{\alpha / 2} v\right\|_{L^{2}(\Omega)}^{2} d \alpha}<\infty \quad \text { s.t. } \quad v(T)=0\right\} .
$$

The problem thus reads as: find $u \in U$ such that $a(u, v)=l(v), \quad \forall v \in V$ where

$$
a(u, v):=\int_{\alpha_{\min }}^{\alpha_{\max }} \phi(\alpha) a^{(\alpha)}(u, v) d \alpha
$$

denotes the distributed bilinear form and $l(v):=(f, v)_{\Omega}$.

3.1. Petrov-Galerkin Method: Modal Expansion. In the Petrov-Galerkin (PG) method, we follow the recent theory of fractional Sturm-Liouville eigen-problems (FSLP) in [61] and employ the corresponding eigenfunctions, known as the Jacobi Poly-fractonomials (of first kind) given in the standard domain $[-1,1]$ by

$$
{ }^{(1)} \mathcal{P}_{n}^{\mu}(\xi)=(1+\xi)^{\mu} P_{n-1}^{-\mu, \mu}(\xi), \quad \xi \in[-1,1],
$$

as non-polynomial basis functions consisting of a fractional term multiplied by the Jacobi polynomial $P_{n-1}^{-\mu, \mu}(\xi)$, hence we employ (3.7) in construction of a basis to formulate a projection type scheme, namely modal expansion. We represent the solution in terms of the elements of the basis space $U_{N}$ given as follows

$$
U_{N}=\operatorname{span}\left\{{ }^{(1)} \mathcal{P}_{n}^{\mu}(\xi), \quad \xi \in[-1,1], \quad n=1,2, \cdots, N\right\}
$$


via the poly-fractonomial modal expansion as

$$
u_{N}(\xi)=\sum_{n=1}^{N} c_{n}^{(1)} \mathcal{P}_{n}^{\mu}(\xi)
$$

in which $\mu$ is to be fixed as a fractional parameter a priori depending on the range of distribution order interval, i.e. $\mu \in(0,1)$ if $\alpha_{\max } \in(0,1]$ and $\mu \in(1,2)$ if $\alpha_{\max } \in(1,2]$. It can also be tunned to capture possible singularities in the exact solution if some knowledge about that is available.

Moreover, in the PG scheme, we employ another space of test functions $V_{N}$, however of the same dimension, given by

$$
V_{N}=\operatorname{span}\left\{{ }^{(2)} \mathcal{P}_{k}^{\mu}(\xi), \quad \xi \in[-1,1], \quad k=1,2, \cdots, N\right\},
$$

in which ${ }^{(2)} \mathcal{P}_{k}^{\mu}(\xi)=(1-\xi)^{\mu} P_{k-1}^{\mu,-\mu}(\xi)$ denotes the Jacobi poly-fractonomial of second kind, which is the explicit eigenfunction of fractional Sturm-Liouville problem of second kind in [61].

It should be noted that since $\phi(\alpha) \geq 0$ and $\int_{\alpha_{\max }}^{\alpha_{\max }} \phi(\alpha) d \alpha=1$ it is not difficult to see that $U_{N} \subset U$ and $V_{N} \subset V$ when $\mu$ is chosen properly. Therefore, the bilinear form (3.3) reduces to the discrete bilinear form

$$
a_{h}^{\alpha}\left(u_{N}, v_{N}\right)=\left({ }_{0} \mathcal{D}_{t}^{\alpha / 2} u_{N},{ }_{t} \mathcal{D}_{T}^{\alpha / 2} v_{N}\right)_{\Omega}
$$

and thus the problem reads as: find $u_{N} \in U_{N}$ such that

$$
a_{h}\left(u_{N}, v_{N}\right)=l_{h}\left(v_{N}\right), \quad \forall v_{N} \in V_{N},
$$

where $a_{h}\left(u_{N}, v_{N}\right):=\int_{\alpha_{\min }}^{\alpha_{\max }} \phi(\alpha) a_{h}^{\alpha}\left(u_{N}, v_{N}\right) d \alpha$ denotes the discrete distributed bilinear form and $l_{h}\left(v_{N}\right):=\left(f, v_{N}\right)_{\Omega}$ represents the load vector.

By substituting the expansion (3.9), choosing $v_{N}={ }^{(2)} \mathcal{P}_{k}^{\mu}(\xi) \in V_{N}, k=1,2, \cdots, N$ and using (2.7), the discrete distributed bilinear form in (3.12) can be written as

$$
\int_{\alpha_{\min }}^{\alpha_{\max }} \phi(\alpha) \sum_{n=1}^{N} c_{n}\left(\frac{2}{T}\right)^{\alpha}\left({ }_{-1} \mathcal{D}_{\xi}^{\alpha / 2}\left[{ }^{(1)} \mathcal{P}_{n}^{\mu}(\xi)\right],{ }_{\xi} \mathcal{D}_{1}^{\alpha / 2}\left[{ }^{(2)} \mathcal{P}_{k}^{\mu}(\xi)\right]\right)_{\Omega} d \alpha
$$

From Lemma 2.1, we have

$$
\begin{aligned}
{ }_{-1} \mathcal{D}_{\xi}^{\alpha / 2}\left[{ }^{(1)} \mathcal{P}_{n}^{\mu}(\xi)\right] & =\frac{\Gamma(n+\mu)}{\Gamma(n+\eta)}{ }^{(1)} \mathcal{P}_{n}^{\eta}(\xi) \\
{ }_{\xi}^{R L} \mathcal{D}_{1}^{\alpha / 2}\left[{ }^{(2)} \mathcal{P}_{k}^{\mu}(\xi)\right], & =\frac{\Gamma(k+\mu)}{\Gamma(k+\eta)}{ }^{(2)} \mathcal{P}_{k}^{\eta}(\xi),
\end{aligned}
$$

where $\eta=\mu-\alpha / 2$. Thus, by changing the order of summation, the integral 3.13 takes the form

$$
\begin{aligned}
& \sum_{n=1}^{N} c_{n} \int_{\alpha_{\min }}^{\alpha_{\max }} \phi(\alpha)\left(\frac{2}{T}\right)^{\alpha} \frac{\Gamma(n+\mu)}{\Gamma(n+\eta)} \frac{\Gamma(k+\mu)}{\Gamma(k+\eta)}\left({ }^{(1)} \mathcal{P}_{n}^{\eta}(\xi),{ }^{(2)} \mathcal{P}_{k}^{\eta}(\xi)\right)_{\Omega} d \alpha \\
= & \sum_{n=1}^{N} c_{n} \int_{\alpha_{\min }}^{\alpha_{\max }} \phi(\alpha)\left(\frac{2}{T}\right)^{\alpha} \frac{\Gamma(n+\mu)}{\Gamma(n+\eta)} \frac{\Gamma(k+\mu)}{\Gamma(k+\eta)} \int_{-1}^{1}{ }^{(1)} \mathcal{P}_{n}^{\eta}(\xi){ }^{(2)} \mathcal{P}_{k}^{\eta}(\xi) d \xi d \alpha,
\end{aligned}
$$


where by changing the order of integrations we get

$$
\begin{aligned}
& a_{h}\left(u_{N}, v_{N}\right) \\
= & \sum_{n=1}^{N} c_{n} \int_{-1}^{1}\left[\int_{\alpha_{\min }}^{\alpha_{\max }} \phi(\alpha)\left(\frac{2}{T}\right)^{\alpha} \frac{\Gamma(n+\mu)}{\Gamma(n+\eta)} \frac{\Gamma(k+\mu)}{\Gamma(k+\eta)}{ }^{(1)} \mathcal{P}_{n}^{\eta}(\xi){ }^{(2)} \mathcal{P}_{k}^{\eta}(\xi) d \alpha\right] d \xi
\end{aligned}
$$

Theorem 3.1 (Spectrally/Exponentially Accurate Quadrature Rule in $\alpha$-Dimension).

Part $A: \forall \xi=\xi_{0} \in[-1,1]$ fixed, and $\forall n \in \mathbb{N} \cup\{0\}$, the Jacobi polynomial $P_{n}^{ \pm \alpha, \mp \alpha}\left(\xi_{0}\right)$ is a polynomial of order $n$ in $\alpha$.

Part B: Let $\phi \in H^{r}\left(\left[\alpha_{\min }, \alpha_{\max }\right]\right), r>0$. Then $\forall \mu \geq \alpha_{\max } / 2$

$$
\begin{aligned}
& \mid \int_{\alpha_{\min }}^{\alpha_{\max }} \phi(\alpha)\left(\frac{2}{T}\right)^{\alpha} \frac{\Gamma(n+\mu)}{\Gamma(n+\eta)} \frac{\Gamma(k+\mu)}{\Gamma(k+\eta)}{ }^{(1)} \mathcal{P}_{n}^{\eta}(\xi){ }^{(2)} \mathcal{P}_{k}^{\eta}(\xi) d \alpha- \\
& \quad \sum_{q=1}^{Q} \widetilde{w}_{q} \phi\left(\alpha_{q}\right)\left(\frac{2}{T}\right)^{\alpha_{q}} \frac{\Gamma(n+\mu)}{\Gamma\left(n+\eta_{q}\right)} \frac{\Gamma(k+\mu)}{\Gamma\left(k+\eta_{q}\right)}{ }^{(1)} \mathcal{P}_{n}^{\eta_{q}}(\xi){ }^{(2)} \mathcal{P}_{k}^{\eta_{q}}(\xi) \mid \leq \mathscr{C} Q^{-r}\|\phi\|_{H^{r}\left(\left[\alpha_{\min }, \alpha_{\max }\right]\right)},
\end{aligned}
$$

where $\mathscr{C}>0, \phi_{N}(\alpha)=\sum_{n=0}^{N} \widetilde{\rho}_{n} P_{n}(\alpha)$ denotes the polynomial expansion of $\phi(\alpha)$, and $\left.\left\{\alpha_{q}, \widetilde{w}_{q}\right\}\right|_{q=1} ^{Q}$ represents the set of Gauss-Legendre quadrature points and weights.

Part C: If $\phi(\alpha)$ is smooth, the quadrature rule in $\alpha$-dimension becomes exponentially accurate in $Q$.

Proof. See Appendix (D).

By theorem (3.1) and performing an affine mapping from $\left[\alpha \in \alpha_{\min }, \alpha_{\max }\right]$ to the standard domain $\alpha_{s t} \in[-1,1]$, the inner integral in (3.17) can be evaluated with spectral accuracy by employing a Gauss-Legendre quadrature rule. Then by changing the order of summation and integral $a_{h}\left(u_{N}, v_{N}\right)=l_{h}\left(v_{N}\right)$ can be written as:

$$
\begin{array}{r}
\sum_{n=1}^{N} \sum_{j=1}^{Q} c_{n} J_{\alpha} w_{j} \phi_{j}\left(\frac{2}{T}\right)^{\alpha_{j}} \frac{\Gamma(n+\mu)}{\Gamma\left(n+\eta_{j}\right)} \frac{\Gamma(k+\mu)}{\Gamma\left(k+\eta_{j}\right)} \int_{-1}^{1}{ }^{(1)} \mathcal{P}_{n}^{\eta_{j}}(\xi){ }^{(2)} \mathcal{P}_{k}^{\eta_{j}}(\xi) d \xi \\
=\left(f,{ }^{(2)} \mathcal{P}_{k}^{\mu}(\xi)\right)_{\Omega}, \quad k=1,2, \ldots, N,
\end{array}
$$

where $J_{\alpha}=\frac{d \alpha}{d \alpha_{s t}}=\frac{\left(\alpha_{\max }-\alpha_{\min }\right)}{2}$ is constant and $\alpha_{j}=\alpha\left(\alpha_{s t_{j}}\right), \phi_{j}=\phi\left(\alpha\left(\alpha_{s t_{j}}\right)\right), \eta_{j}=\mu-\alpha_{j} / 2$ and $\alpha_{s t_{j}}$ and $w_{j}$ are the quadrature points and weights respectively. The linear system is then constructed as

$$
\mathcal{S} \vec{c}=\overrightarrow{\mathcal{F}},
$$

in which the entries of the stiffness matrix $\mathcal{S}$ and force vector $\overrightarrow{\mathcal{F}}$ are given by

$$
\mathcal{S}_{k n}=\sum_{j=0}^{Q-1} J_{\alpha} w_{j} \phi_{j}\left(\frac{2}{T}\right)^{\alpha_{j}} C_{k n} \int_{-1}^{1}{ }^{(1)} \mathcal{P}_{n}^{\eta_{j}}(\xi)^{(2)} \mathcal{P}_{k}^{\eta_{j}}(\xi) d \xi
$$

and

$$
\mathcal{F}_{k}=\int_{-1}^{1} f(\xi)^{(2)} \mathcal{P}_{k}^{\mu}(\xi) d \xi
$$


respectively, where

$$
C_{k n} \equiv \frac{\Gamma(n+\mu)}{\Gamma\left(n+\eta_{j}\right)} \frac{\Gamma(k+\mu)}{\Gamma\left(k+\eta_{j}\right)}
$$

REMARK 3.1. For each fixed $j$ and given the structure of ${ }^{(1)} \mathcal{P}_{n}^{\eta_{j}}(\xi)$ and ${ }^{(2)} \mathcal{P}_{k}^{\eta_{j}}(\xi)$, the above integrations take the form

$$
\begin{aligned}
\int_{-1}^{1}{ }^{(1)} \mathcal{P}_{n}^{\eta_{j}}(\xi){ }^{(2)} \mathcal{P}_{k}^{\eta_{j}}(\xi) d \xi & =\int_{-1}^{1}(1-\xi)^{\eta_{j}}(1+\xi)^{\eta_{j}} P_{k-1}^{\eta_{j},-\eta_{j}}(\xi) P_{n-1}^{-\eta_{j}, \eta_{j}}(\xi) d \xi \\
\int_{-1}^{1} f(\xi)^{(2)} \mathcal{P}_{k}^{\mu}(\xi) d \xi & =\int_{-1}^{1}(1-\xi)^{\mu} f(\xi) P_{k-1}^{\mu,-\mu}(\xi) d \xi
\end{aligned}
$$

and therefore, the full stiffness matrix $\mathcal{S}$ and vector $\overrightarrow{\mathcal{F}}$ can be constructed accurately using a proper Gauss-Lobatto-Jacobi rule corresponding to the weight function $(1-\xi)^{\eta_{j}}(1+\xi)^{\eta_{j}}$ and $(1-\xi)^{\eta_{j}}$ respectively.

3.2. Discrete Stability Analysis. In this section, we investigate the stability of the numerical scheme, developed based on the aforementioned choice of solution and test function space considering the bilinear form in (3.12).

THEOREM 3.2. The scheme (3.12) is stable and the following inequality holds

$$
\inf _{u_{N} \in U_{N} v_{N} \in V_{N}} \sup _{\left\|u_{N}\right\|_{U_{N}}\left\|v_{N}\right\|_{V_{N}}} \geq \beta .
$$

Proof. Recalling from 3.6

$$
a_{h}\left(u_{N}, v_{N}\right)=\int_{\alpha_{\min }}^{\alpha_{\max }} \phi(\alpha) a_{h}^{\alpha}\left(u_{N}, v_{N}\right) d \alpha
$$

where, by lemma (2.1),

$$
\begin{aligned}
a_{h}^{\alpha}\left(u_{N}, v_{N}\right) & =a_{h}^{\alpha}\left(\sum_{n=1}^{N} a_{n}{ }^{(1)} \mathcal{P}_{n}^{\mu}(\xi), \sum_{k=1}^{N} a_{k}{ }^{(2)} \mathcal{P}_{k}^{\mu}(\xi)\right), \quad \forall \alpha \in\left(\alpha_{\min }, \alpha_{\max }\right), \\
& =\sum_{n=1}^{N} \sum_{k=1}^{N} a_{n} a_{k} C_{n, k}^{\mu, \alpha} \int_{-1}^{1}(1-\xi)^{\tilde{\mu}}(1+\xi)^{-\tilde{\mu}}(1+\xi)^{2 \tilde{\mu}} P_{k-1}^{\tilde{\mu},-\tilde{\mu}}(\xi) P_{n-1}^{-\tilde{\mu}, \tilde{\mu}}(\xi) d \xi
\end{aligned}
$$

in which, $\tilde{\mu}=\mu-\alpha / 2, C_{n, k}^{\mu, \alpha}=\frac{\Gamma(n+\mu)}{\Gamma(n+\tilde{\mu})} \frac{\Gamma(k+\mu)}{\Gamma(k+\tilde{\mu})}$ and $(1+\xi)^{\tilde{\mu}}$ is replaced by $(1+\xi)^{-\tilde{\mu}}(1+\xi)^{2 \tilde{\mu}}$. We let $\tilde{\mu}>-1 / 2$, hence the function $(1+\xi)^{2 \tilde{\mu}}$ is nonnegative, nondecreasing, continuous and integrable in the integration domain. Therefore,

$$
a_{h}^{\alpha}\left(u_{N}, v_{N}\right) \geq \sum_{n=1}^{N} \sum_{k=1}^{N} a_{n} a_{k} C_{n, k}^{\mu, \alpha} C_{0} \int_{-1}^{1}(1-\xi)^{\tilde{\mu}}(1+\xi)^{-\tilde{\mu}} P_{k-1}^{\tilde{\mu},-\tilde{\mu}}(\xi) P_{n-1}^{-\tilde{\mu}, \tilde{\mu}}(\xi) d \xi
$$

Moreover, the Jacobi polynomial $P_{n-1}^{-\tilde{\mu}, \tilde{\mu}}(\xi)$ can be expanded as:

$$
P_{n-1}^{-\tilde{\mu} \tilde{\mu}}(\xi)=\sum_{j=0}^{n-1}\left(\begin{array}{c}
n-1+j \\
j
\end{array}\right)\left(\begin{array}{c}
n-1+\tilde{\mu} \\
n-1-j
\end{array}\right)(-1)^{j-n+1}\left(\frac{1}{2}\right)^{j}(1+\xi)^{j} .
$$


By multiplying and dividing each term within the summation by $\left(\begin{array}{c}n-1-\tilde{\mu} \\ n-1-j\end{array}\right)$ we get

$$
P_{n-1}^{-\tilde{\mu}, \tilde{\mu}}(\xi)=\sum_{j=0}^{n-1} \mathcal{A}_{n, j}^{\tilde{\mu}}\left(\begin{array}{c}
n-1+j \\
j
\end{array}\right)\left(\begin{array}{c}
n-1-\tilde{\mu} \\
n-1-j
\end{array}\right)(-1)^{j-n+1}\left(\frac{1}{2}\right)^{j}(1+\xi)^{j},
$$

where $\mathcal{A}_{n, j}^{\tilde{\mu}}=\left(\begin{array}{c}n-1+\tilde{\mu} \\ n-1-j\end{array}\right) /\left(\begin{array}{c}n-1-\tilde{\mu} \\ n-1-j\end{array}\right)$ is nondecreasing, positive and bounded $\forall n, j, \tilde{\mu}$. Therefore, there exists $C_{1}=C_{1}(n)>0$ such that

$$
\begin{aligned}
a_{h}^{\alpha}\left(u_{N}, v_{N}\right) & \geq \sum_{n=1}^{N} \sum_{k=1}^{N} a_{n} a_{k} C_{n, k}^{\mu, \alpha} C_{0} C_{1}(n) \int_{-1}^{1}(1-\xi)^{\tilde{\mu}}(1+\xi)^{-\tilde{\mu}} P_{k-1}^{\tilde{\mu},-\tilde{\mu}}(\xi) P_{n-1}^{\tilde{\mu},-\tilde{\mu}}(\xi) d \xi \\
& \geq C_{0} \sum_{k=1}^{N} a_{k}^{2} C_{k, k}^{\mu, \alpha} C_{1}(n) \epsilon_{k-1}^{\tilde{\mu},-\tilde{\mu}}
\end{aligned}
$$

in which $\epsilon_{k-1}^{\tilde{\mu}, \tilde{\mu}}=\frac{2}{2 k-1} \frac{\Gamma(k+\mu) \Gamma(k-\mu)}{(k-1) ! \Gamma(k)}$. Hence,

$$
a_{h}\left(u_{N}, v_{N}\right) \geq C_{0} \int_{\alpha_{\min }}^{\alpha_{\max }} \phi(\alpha) \sum_{k=1}^{N} a_{k}^{2} C_{k, k}^{\mu, \alpha} C_{1}(n) \epsilon_{k-1}^{\tilde{\mu},-\tilde{\mu}} d \alpha
$$

Moreover, we have

$$
\left\|v_{N}\right\|_{V_{N}}^{2}=\int_{\alpha_{\min }}^{\alpha_{\max }} \phi(\alpha)\left\|_{-1} \mathcal{D}_{x}^{\alpha / 2} v_{N}\right\|_{L^{2}(\Omega)}^{2} d \alpha
$$

where by considering $v_{n}=\sum_{k=1}^{N} a_{k}{ }^{(2)} \mathcal{P}_{k}^{\mu}(\xi)$, we can write $\forall \alpha \in\left(\alpha_{\min }, \alpha_{\max }\right)$,

$$
\begin{aligned}
\left\|_{-1} \mathcal{D}_{x}^{\alpha / 2} v_{N}\right\|_{L^{2}(\Omega)}^{2} & =\int_{-1}^{1}\left(\sum_{k=1}^{N} a_{k} \frac{\Gamma(k+\mu)}{\Gamma(k+\tilde{\mu})}(1-\xi)^{\tilde{\mu}} P_{k-1}^{\tilde{\mu},-\tilde{\mu}}(\xi)\right)^{2} d \xi, \\
& \leq \int_{-1}^{1} \sum_{k=1}^{N} a_{k}^{2}\left(\frac{\Gamma(k+\mu)}{\Gamma(k+\tilde{\mu})}\right)^{2}(1-\xi)^{2 \tilde{\mu}}\left(P_{k-1}^{\tilde{\mu},-\tilde{\mu}}(\xi)\right)^{2} d \xi,
\end{aligned}
$$

(By Jensen Inequality).

By multiplying the integrand by $(1+\xi)^{-\tilde{\mu}}(1+\xi)^{\tilde{\mu}}$ and changing the order of summation and integration, we obtain

$$
\begin{aligned}
\left\|_{-1} \mathcal{D}_{x}^{\alpha / 2} v_{N}\right\|_{L^{2}(\Omega)}^{2} & \leq \sum_{k=1}^{N} a_{k}^{2}\left(\frac{\Gamma(k+\mu)}{\Gamma(k+\tilde{\mu})}\right)^{2} \int_{-1}^{1}(1-\xi)^{\tilde{\mu}}(1+\xi)^{-\tilde{\mu}}\left(1-\xi^{2}\right)^{\tilde{\mu}} P_{k-1}^{\tilde{\mu}, \tilde{\mu}}(\xi) P_{k-1}^{\tilde{\mu},-\tilde{\mu}}(\xi) d \xi \\
& \leq \sum_{k=1}^{N} a_{k}^{2}\left(\frac{\Gamma(k+\mu)}{\Gamma(k+\tilde{\mu})}\right)^{2} C_{2} \int_{-1}^{1}(1-\xi)^{\tilde{\mu}}(1+\xi)^{-\tilde{\mu}} P_{k-1}^{\tilde{\mu},-\tilde{\mu}}(\xi) P_{k-1}^{\tilde{\mu},-\tilde{\mu}}(\xi) d \xi
\end{aligned}
$$

since $\tilde{\mu}>-1 / 2$ and consequently $\left(1-\xi^{2}\right)^{\tilde{\mu}}$ is a nonnegative and integrable in the domain of integration. By the orthogonality of Jacobi polynomials, we get

$$
\left\|_{-1} \mathcal{D}_{x}^{\alpha / 2} v_{N}\right\|_{L^{2}(\Omega)}^{2} \leq C_{2} \sum_{k=1}^{N} a_{k}^{2}\left(\frac{\Gamma(k+\mu)}{\Gamma(k+\tilde{\mu})}\right)^{2} \epsilon_{k-1}^{\tilde{\mu},-\tilde{\mu}},
$$


and thus

$$
\left\|v_{N}\right\|_{V_{N}} \leq \sqrt{C_{2} \int_{\alpha_{\min }}^{\alpha_{\max }} \phi(\alpha) \sum_{k=1}^{N} a_{k}^{2}\left(\frac{\Gamma(k+\mu)}{\Gamma(k+\tilde{\mu})}\right)^{2} \epsilon_{k-1}^{\tilde{\mu}-\tilde{\mu}} d \alpha .}
$$

Similarly for $\left\|u_{N}\right\|_{U_{N}}^{2}$ :

$$
\left\|u_{N}\right\|_{U_{N}}^{2}=\int_{\alpha_{\min }}^{\alpha_{\max }} \phi(\alpha)\left\|_{-1} \mathcal{D}_{x}^{\alpha / 2} u_{N}\right\|_{L^{2}(\Omega)}^{2} d \alpha,
$$

where $\forall \alpha \in\left(\alpha_{\min }, \alpha_{\max }\right)$ :

$$
\begin{aligned}
\left\|_{-1} \mathcal{D}_{x}^{\alpha / 2} u_{N}\right\|_{L^{2}(\Omega)}^{2} & =\int_{-1}^{1}\left(\sum_{n=1}^{N} a_{n} \frac{\Gamma(n+\mu)}{\Gamma(n+\tilde{\mu})}(1+\xi)^{\tilde{\mu}} P_{n-1}^{-\tilde{\mu}, \tilde{\mu}}(\xi)\right)^{2} d \xi, \\
& \leq \int_{-1}^{1} \sum_{n=1}^{N} a_{n}^{2}\left(\frac{\Gamma(n+\mu)}{\Gamma(n+\tilde{\mu})}\right)^{2}(1+\xi)^{2 \tilde{\mu}}\left(P_{n-1}^{-\tilde{\mu}, \tilde{\mu}}(\xi)\right)^{2} d \xi,
\end{aligned}
$$

By Jensen Inequality.

Following similar steps, and by multiplying the integrand by $(1-\xi)^{-\tilde{\mu}}(1-\xi)^{\tilde{\mu}}$ and changing the order of summation and integration, we obtain

$$
\begin{aligned}
& \left\|_{-1} \mathcal{D}_{x}^{\alpha / 2} u_{N}\right\|_{L^{2}(\Omega)}^{2}, \\
\leq & \sum_{n=1}^{N} a_{n}^{2}\left(\frac{\Gamma(n+\mu)}{\Gamma(n+\tilde{\mu})}\right)^{2} \int_{-1}^{1}(1-\xi)^{-\tilde{\mu}}(1+\xi)^{\tilde{\mu}}\left(1-\xi^{2}\right)^{\tilde{\mu}} P_{n-1}^{-\tilde{\mu}, \tilde{\mu}}(\xi) P_{n-1}^{-\tilde{\mu}, \tilde{\mu}}(\xi) d \xi, \\
\leq & \sum_{n=1}^{N} a_{n}^{2}\left(\frac{\Gamma(n+\mu)}{\Gamma(n+\tilde{\mu})}\right)^{2} C_{3} \int_{-1}^{1}(1-\xi)^{-\tilde{\mu}}(1+\xi)^{\tilde{\mu}} P_{n-1}^{-\tilde{\mu}, \tilde{\mu}}(\xi) P_{n-1}^{-\tilde{\mu}, \tilde{\mu}}(\xi) d \xi,
\end{aligned}
$$

since $\left(1-\xi^{2}\right)^{\tilde{\mu}}$ is a nonnegative and integrable in the domain of integral. Next, by the orthogonality of Jacobi polynomials,

$$
\left\|_{-1} \mathcal{D}_{x}^{\alpha / 2} u_{N}\right\|_{L^{2}(\Omega)}^{2} \leq C_{3} \sum_{n=1}^{N} a_{n}^{2}\left(\frac{\Gamma(n+\mu)}{\Gamma(n+\tilde{\mu})}\right)^{2} \epsilon_{n-1}^{-\tilde{\mu}, \tilde{\mu}} .
$$

Therefore,

$$
\left\|u_{N}\right\|_{U_{N}} \leq \sqrt{C_{3} \int_{\alpha_{\min }}^{\alpha_{\max }} \phi(\alpha) \sum_{n=1}^{N} a_{n}^{2}\left(\frac{\Gamma(n+\mu)}{\Gamma(n+\tilde{\mu})}\right)^{2} \epsilon_{n-1}^{\tilde{\mu},-\tilde{\mu}} d \alpha},
$$

where $\epsilon_{n-1}^{-\tilde{\mu}, \tilde{\mu}}=\epsilon_{n-1}^{\tilde{\mu},-\tilde{\mu}}$.

Therefore, using (3.22), (3.23) and (3.24),

$$
\inf _{u_{N} \in U_{N} v_{N} \in V_{N}} \sup _{\left\|u_{N}\right\|_{U_{N}}\left\|v_{N}\right\|_{V_{N}}} \geq \frac{a_{0}\left(u_{N}, v_{1_{\min }}\right.}{\sqrt{C_{2} C_{3}}}=\beta .
$$

0 
3.3. Projection Error Analysis. In this section, we investigate the error due to the projection of the true solution onto the defined set of basis functions.

THEOREM 3.3. Let $\frac{d^{r} u}{d t^{r}} \in U$, that is, $\int_{\alpha_{\min }}^{\alpha \max } \phi(\alpha)\left\|_{0} \mathcal{D}_{t}^{r+\alpha / 2} u\right\|_{L^{2}(\Omega)}^{2} d \alpha<\infty$ and $u_{N}$ denotes the projection of the exact solution $u$. Then,

$$
\left\|u-u_{N}\right\|_{U}^{2} \leq C N^{-2 r} \int_{\alpha_{\min }}^{\alpha_{\max }} \phi(\alpha)\left\|_{0} \mathcal{D}_{t}^{r+\alpha / 2} u\right\|_{L^{2}(\Omega)}^{2} d \alpha .
$$

Proof. By performing an affine mapping from $t \in[0, T]$ to the standard domain $\xi \in$ $[-1,1]$, we expand the exact solution $u$ in terms of the following infinite series of Jacobi poly-fractonomials

$$
u(\xi)=\sum_{n=1}^{\infty} c_{n}{ }^{(1)} \mathcal{P}_{n}^{\mu}(\xi)
$$

Then, we note that by using (2.1) and (2.7),

$$
\begin{aligned}
{ }_{0} \mathcal{D}_{t}^{r+\alpha / 2} u(\xi(t)) & =\left(\frac{2}{T}\right)^{r+\alpha / 2}{ }_{-1} \mathcal{I}_{\xi}^{\mu-\alpha / 2} \frac{d^{r}}{d \xi^{r}}-1 \mathcal{D}_{\xi}^{\mu} u(\xi), \\
& =\left(\frac{2}{T}\right)^{r+\alpha / 2} \sum_{n=1}^{\infty} c_{n}\left(\frac{\Gamma(n+\mu)}{\Gamma(n)}\right)_{-1} \mathcal{I}_{\xi}^{\mu-\alpha / 2} \frac{d^{r}}{d \xi^{r}}\left[P_{n-1}(\xi)\right],
\end{aligned}
$$

where,

$$
\frac{d^{r}}{d \xi^{r}}\left[P_{n-1}(\xi)\right]= \begin{cases}\frac{(n-1+r) !}{2 r(n-1) !} P_{n-1-r}^{r, r}(\xi) & r<n \\ 0 & r \geq n\end{cases}
$$

Thus, by multiplying with a proper weight function, $w(\xi)=(1+\xi)^{r / 2-\mu+\alpha / 2}(1-\xi)^{r / 2}$, the right-hand-side of (3.25) takes the form

$$
\begin{aligned}
\int_{\alpha_{\min }}^{\alpha_{\max }} \phi(\alpha)\left(\frac{2}{T}\right)^{2 r+\alpha / 2} & \|(1+\xi)^{r / 2-\mu+\alpha / 2}(1-\xi)^{r / 2} \\
& \sum_{n=r+1}^{\infty} c_{n}\left(\frac{\Gamma(n+\mu)}{\Gamma(n)}\right) \frac{(n-1+r) !}{2 r(n-1) !}{ }_{-1} I_{\xi}^{\mu-\alpha / 2} P_{n-1-r}^{r, r}(\xi) \|_{L^{2}(\Omega)}^{2} d \alpha .
\end{aligned}
$$

By expanding the Jacobi polynomial as

$$
P_{n-1-r}^{r, r}(\xi)=(-1)^{n-1-r} \sum_{j=0}^{n-1-r}\left(\begin{array}{c}
n-1+r+j \\
j
\end{array}\right)\left(\begin{array}{c}
n-1 \\
n-1-r-j
\end{array}\right)\left(\frac{-1}{2}\right)^{j}(1+\xi)^{j},
$$

and changing the order of summation and the integration, we obtain the fractional integral as

$$
\begin{aligned}
& -\mathcal{I}_{\xi}^{\mu-\alpha / 2} P_{n-1-r}^{r, r}(\xi), \\
& =(-1)^{n-1-r}(1+\xi)^{\mu-\alpha / 2} \sum_{j=0}^{n-1-r}\left(\begin{array}{c}
n-1+r+j \\
j
\end{array}\right)\left(\begin{array}{c}
n-1 \\
n-1-r-j
\end{array}\right)\left(\frac{-1}{2}\right)^{j} \frac{\Gamma(1+j)}{\Gamma(1+j+\mu-\alpha / 2)}(1+\xi)^{j}, \\
& =(-1)^{n-1-r}(1+\xi)^{\mu-\alpha / 2} \sum_{q=0}^{n-1-r} \tilde{c}_{q}(\alpha) P_{q}^{r, r}(\xi),
\end{aligned}
$$


where, the coefficient, $\tilde{c}_{q}(\alpha)$, can be obtained using the orthogonality of Jacobi polynomials.

Hence, by taking $C_{n}=c_{n}(-1)^{n-1} \frac{\Gamma(n+\mu)}{\Gamma(n)}$, 3.27) takes the form

$$
\begin{aligned}
& \int_{\alpha_{\min }}^{\alpha_{\max }} \phi(\alpha)\left(\frac{2}{T}\right)^{2 r+\alpha}\left\|(1+\xi)^{r / 2}(1-\xi)^{r / 2} \sum_{n=r+1}^{\infty} C_{n} \frac{(n-1+r) !}{2 r(n-1) !}(-1)^{-r} \sum_{q=0}^{n-1-r} \tilde{c}_{q}(\alpha) P_{q}^{r, r}(\xi)\right\|_{L^{2}(\Omega)}^{2} d \alpha, \\
= & \int_{\alpha_{\min }}^{\alpha_{\max }} \phi(\alpha)\left(\frac{2}{T}\right)^{2 r+\alpha}\left[\int_{-1}^{1}(1+\xi)^{r}(1-\xi)^{r}\left(\sum_{n=r+1}^{\infty} C_{n} \frac{(n-1+r) !}{2 r(n-1) !} \sum_{q=0}^{n-1-r} \tilde{c}_{q}(\alpha) P_{q}^{r, r}(\xi)\right)^{2} d \xi\right] d \alpha .
\end{aligned}
$$

Then, we change the order of two summations in order to use the orthogonality of Jacobi polynomials and obtain

$$
\begin{aligned}
& \int_{\alpha_{\min }}^{\alpha_{\max }} \phi(\alpha)\left(\frac{2}{T}\right)^{2 r+\alpha}\left[\int_{-1}^{1}(1+\xi)^{r}(1-\xi)^{r}\left(\sum_{q=0}^{\infty} \sum_{n=r+1+q}^{\infty} C_{n} \frac{(n-1+r) !}{2 r(n-1) !} \tilde{c}_{q}(\alpha) P_{q}^{r, r}(\xi)\right)^{2} d \xi\right] d \alpha, \\
= & \int_{\alpha_{\min }}^{\alpha_{\max }} \phi(\alpha)\left(\frac{2}{T}\right)^{2 r+\alpha}\left[\int_{-1}^{1}(1+\xi)^{r}(1-\xi)^{r} \sum_{q=0}^{\infty}\left(\sum_{n=r+1+q}^{\infty} C_{n} \frac{(n-1+r) !}{2 r(n-1) !}\right)^{2} \tilde{c}_{q}^{2}(\alpha)\left(P_{q}^{r, r}(\xi)\right)^{2} d \xi\right] d \alpha, \\
= & \sum_{q=0}^{\infty}\left(\sum_{n=r+1+q}^{\infty} C_{n} \frac{(n-1+r) !}{2 r(n-1) !}\right)^{2} \int_{-1}^{1}(1+\xi)^{r}(1-\xi)^{r}\left(P_{q}^{r, r}(\xi)\right)^{2} d \xi \int_{\alpha_{\min }}^{\alpha_{\max }} \phi(\alpha)\left(\frac{2}{T}\right)^{2 r+\alpha} \tilde{c}_{q}^{2}(\alpha) d \alpha, \\
= & \sum_{q=0}^{\infty}\left(\sum_{n=r+1+q}^{\infty} C_{n} \frac{(n-1+r) !}{2 r(n-1) !}\right)^{2} \frac{2^{2 r+1}}{2 q+2 r+1} \frac{((q+1) !)^{2}}{q !(q+2 r) !} \int_{\alpha_{\min }}^{\alpha_{\max }} \phi(\alpha)\left(\frac{2}{T}\right)^{2 r+\alpha} \tilde{c}_{q}^{2}(\alpha) d \alpha, \\
= & \frac{2^{2 r+1}\left(\frac{2}{T}\right)^{2 r}}{(2 r)^{2}} \sum_{q=0}^{\infty}\left(\sum_{n=r+1+q}^{\infty} C_{n} \frac{(n-1+r) !}{(n-1) !}\right)^{2} \frac{(q+1)^{2} q !}{(2 q+2 r+1)(q+2 r) !} \int_{\alpha_{\min }}^{\alpha_{\max }} \phi(\alpha)\left(\frac{2}{T}\right)^{\alpha} \tilde{c}_{q}^{2}(\alpha) d \alpha .
\end{aligned}
$$

Moreover, using the approximation of the solution given in (3.9) and by multiplying with the proper weight functions, the left-hand-side of (3.25) takes the form

$$
\begin{aligned}
& \left\|(1+\xi)^{-\mu+\alpha / 2}\left(u-u_{N}\right)\right\|_{U}^{2}, \\
& =\int_{\alpha_{\min }}^{\alpha_{\max }} \phi(\alpha)\left(\frac{2}{T}\right)^{\alpha}\left\|(1+\xi)^{-\mu+\alpha / 2} \sum_{n=N+1}^{\infty} c_{n}\left(\frac{\Gamma(n+\mu)}{\Gamma(n)}\right){ }_{-1} \mathcal{I}_{\xi}^{\mu-\alpha / 2} P_{n-1}(\xi)\right\|_{L^{2}(\Omega)}^{2} d \alpha,
\end{aligned}
$$

in which, ${ }_{-1} \mathcal{D}_{\xi}^{\alpha / 2}={ }_{-1} \mathcal{D}_{\xi}^{\alpha / 2-\mu+\mu}={ }_{-1} \mathcal{I}_{\xi}^{\mu-\alpha / 2}{ }_{-1} \mathcal{D}_{\xi}^{\mu}$ and the fractional derivative is taken using 2.1. By expanding the Legendre polynomial as

$$
P_{n-1}(\xi)=(-1)^{n-1} \sum_{j=0}^{n-1}\left(\begin{array}{c}
n-1+j \\
j
\end{array}\right)\left(\begin{array}{c}
n-1 \\
n-1-j
\end{array}\right)\left(\frac{-1}{2}\right)^{j}(1+\xi)^{j}
$$

and following similar steps as in (3.28), we obtain the fractional integral as

$$
{ }_{-1} I_{\xi}^{\mu-\alpha / 2} P_{n-1}(\xi)=(-1)^{n-1}(1+\xi)^{\mu-\alpha / 2} \sum_{q=0}^{n-1} \tilde{a}_{q}(\alpha) P_{q}(\xi)
$$


where the coefficient, $\tilde{a}_{q}(\alpha)$, can be obtained using the orthogonality of Legendre polynomials. Hence, (3.31) takes the form

$$
\left\|(1+\xi)^{-\mu+\alpha / 2}\left(u-u_{N}\right)\right\|_{U}^{2}=\int_{\alpha_{\min }}^{\alpha_{\max }} \phi(\alpha)\left(\frac{2}{T}\right)^{\alpha} \int_{-1}^{1}\left(\sum_{n=N+1}^{\infty} C_{n} \sum_{q=0}^{n-1} \tilde{a}_{q}(\alpha) P_{q}(\xi)\right)^{2} d \xi d \alpha
$$

in which, $C_{n}=c_{n}(-1)^{n-1} \frac{\Gamma(n+\mu)}{\Gamma(n)}$. We change the order of two summations to use the orthogonality of Legendre polynomials and obtain

$$
\begin{aligned}
& \int_{\alpha_{\min }}^{\alpha_{\max }} \phi(\alpha)\left(\frac{2}{T}\right)^{\alpha} \int_{-1}^{1}\left(\sum_{q=0}^{N} \sum_{n=q+1}^{\infty} C_{n} \tilde{a}_{q}(\alpha) P_{q}(\xi)+\sum_{q=N+1}^{\infty} \sum_{n=q+1}^{\infty} C_{n} \tilde{a}_{q}(\alpha) P_{q}(\xi)\right)^{2} d \xi d \alpha, \\
& =\int_{\alpha_{\min }}^{\alpha_{\max }} \phi(\alpha)\left(\frac{2}{T}\right)^{\alpha}\left(\sum_{q=0}^{N}\left(\sum_{n=q+1}^{\infty} C_{n}\right)^{2} \tilde{a}_{q}^{2}(\alpha) \int_{-1}^{1}\left(P_{q}(\xi)\right)^{2} d \xi+\sum_{q=N+1}^{\infty}\left(\sum_{n=q+1}^{\infty} C_{n}\right)^{2} \tilde{a}_{q}^{2}(\alpha) \int_{-1}^{1}\left(P_{q}(\xi)\right)^{2} d \xi\right) d \alpha, \\
& \leq \sum_{q=N+1}^{\infty}\left(\sum_{n=q+1}^{\infty} C_{n}\right)^{2} \frac{2}{2 q+1} \int_{\alpha_{\min }}^{\alpha_{\max }} \phi(\alpha)\left(\frac{2}{T}\right)^{\alpha} \tilde{a}_{q}^{2}(\alpha) d \alpha, \\
& \leq\left(\frac{N !}{(N-r) !}\right)^{2} \sum_{q=N+1}^{\infty}\left(\sum_{n=q+1}^{\infty} C_{n} \frac{(n-1+r) !}{(n-1) !}\right)^{2} \frac{2}{2 q+1} \frac{(q+1)^{2} q !}{(2 q+2 r+1)(q+2 r) !} \int_{\alpha_{\min }}^{\alpha_{\max }} \phi(\alpha)\left(\frac{2}{T}\right)^{\alpha} \tilde{a}_{q}^{2}(\alpha) d \alpha, \\
& \leq\left(\frac{N !}{(N-r) !}\right)^{2} \int_{\alpha_{\min }}^{\alpha_{\max }} \phi(\alpha)\left(\frac{2}{T}\right)^{2 r+\alpha}\left\|(1+\xi)^{r / 2-\mu+\alpha / 2}(1-\xi)^{r / 2}{ }_{-1} \mathcal{D}_{\xi}^{r+\alpha / 2} u\right\|_{L^{2}(\Omega)}^{2} d \alpha, \\
& \leq\left(\frac{N !}{(N-r) !}\right)^{2} \int_{\alpha_{\min }}^{\alpha_{\max }} \phi(\alpha)\left\|_{0} \mathcal{D}_{t}^{r+\alpha / 2} u\right\|_{L^{2}(\Omega)}^{2} d \alpha .
\end{aligned}
$$

Therefore,

$$
\left\|u-u_{N}\right\|_{U}^{2} \leq\left\|(1+\xi)^{-\mu+\alpha / 2}\left(u-u_{N}\right)\right\|_{U}^{2} \leq C N^{-2 r} \int_{\alpha_{\min }}^{\alpha_{\max }} \phi(\alpha)\left\|_{0} \mathcal{D}_{t}^{r+\alpha / 2} u\right\|_{L^{2}(\Omega)}^{2} d \alpha .
$$

REMARK 3.2. Since the inf-sup condition holds (see Theorem (3.2)), by the Banach-NečasBabuška theorem [15], the error in the numerical scheme is less that or equal to a constant times the projection error. Choosing the projection $u_{N}$ in Theorem (3.3), we infer the spectral accuracy of the scheme.

4. Fractional Collocation Method: Nodal Expansion. Next, we represent the solution via the following poly-fractonomial nodal expansion as

$$
u_{N}(\xi)=\sum_{j=1}^{N} u_{N}\left(\xi_{j}\right) h_{j}^{\mu}(\xi)
$$

where $h_{j}^{\mu}(\xi)$ represent fractional Lagrange interpolants FLIs, which are all of fractional order $(N+\mu-1)$ and constructed using the aforementioned interpolations points $-1=\xi_{1}<\xi_{2}<$ $\cdots<\xi_{N}=1$ as:

$$
h_{j}^{\mu}(\xi)=\left(\frac{\xi-x_{1}}{x_{j}-x_{1}}\right)^{\mu} \prod_{\substack{k=1 \\ k \neq j}}^{N}\left(\frac{\xi-x_{k}}{x_{j}-x_{k}}\right), \quad j=2,3, \cdots, N .
$$


Because of the homogeneous Dirichlet boundary condition(s) in 2.27) and 2.28), $u_{N}(-1)=$ 0 , and thus we only construct $h_{j}^{\mu}(\xi)$ for $j=2,3, \cdots, N$. We note that FLIs satisfy the Kronecker delta property, i.e., $h_{j}^{\mu}\left(\xi_{k}\right)=\delta_{j k}$, at interpolation points, however they vary as a polyfractonomial between $\xi_{k}$ 's.

4.1. Differentiation Matrices $\mathbf{D}^{\alpha}$ and $\mathbf{D}^{1+\alpha}, \alpha \in(0,1)$. By breaking the domain of integration in $\alpha, 2.25$ takes the form

$$
\int_{\alpha_{\min }}^{1} \phi(\alpha)_{0} \mathcal{D}_{t}^{\alpha} u(t) d \alpha+\int_{1}^{\alpha_{\max }} \phi(\alpha)_{0} \mathcal{D}_{t}^{\alpha} u(t) d \alpha=f(t ; u), \quad \forall t \in(0, T] .
$$

Following [64], we obtain the corresponding fractional differentiation matrices $\mathbf{D}^{\alpha}$ and $\mathbf{D}^{1+\alpha}$, $\alpha \in(0,1)$ by substituting (4.2) in (4.1) and taking the $\alpha$-th order fractional derivative. These matrices are given as:

$$
\mathbf{D}_{i j}^{\alpha}=\frac{1}{\left(\xi_{j}+1\right)^{\mu}} \sum_{n=1}^{N} \beta_{n}^{j} \sum_{q=\lceil\alpha-\mu\rceil}^{n-1} b_{n q}\left(\xi_{i}+1\right)^{q+\mu-\alpha},
$$

and

$$
\mathbf{D}_{i j}^{1+\alpha}=\frac{1}{\left(\xi_{j}+1\right)^{\mu}}\left[\sum_{n=1}^{N} \beta_{n}^{j} \sum_{q=\lceil\alpha-\mu\rceil}^{n-1} b_{n q}(q+\mu-\alpha)\left(\xi_{i}+1\right)^{q+\mu-\alpha-1}\right],
$$

in which $\lceil\alpha-\mu\rceil$ denotes the ceiling of $\alpha-\mu$ and

$$
b_{n q}=(-1)^{n+q-1}\left(\frac{1}{2}\right)^{q}\left(\begin{array}{c}
n-1+q \\
q
\end{array}\right)\left(\begin{array}{l}
n-1+\mu \\
n-1-q
\end{array}\right) \frac{\Gamma(q+\mu+1)}{\Gamma(q+\mu-\alpha+1)} .
$$

The coefficients, $\beta_{n}^{j}$, are the coefficients in expansion of the polynomial $p_{j}(\xi)=\prod_{\substack{k=1 \\ k \neq j}}^{N}\left(\frac{\xi-\xi_{k}}{\xi_{j}-\xi_{k}}\right)$ in terms of Jacobi polynomials as

$$
\prod_{\substack{k=1 \\ k \neq j}}^{N}\left(\frac{\xi-\xi_{k}}{x_{j}-x_{k}}\right)=\sum_{n=1}^{N} \beta_{n}^{j} P_{n-1}^{-\mu, \mu}(\xi)
$$

Due to the orthogonality of the Jacobi poly-fractonomials $P_{n-1}^{-\mu, \mu}(\xi)$ with respect to the weight function $w(\xi)=(1-\xi)^{-\mu}(1+\xi)^{-\mu}$, these coefficients can be computed efficiently only once by employing a proper Guass-Lobatto-Jacobi quadrature rule.

Therefore, by substituting the nodal expansion (4.2) into 4.3), performing an affine mapping from $\left[\alpha_{\min }, \alpha_{\max }\right]$ to the standard domain $[-1,1]$, and employing a proper quadrature rule in $\alpha$-domain, 4.3 can be written as

$$
\begin{aligned}
& \sum_{j=2}^{N}\left[\sum_{q}^{Q} w_{q} \phi\left(\alpha_{q}\right)\left(\frac{2}{T}\right)^{\alpha_{q}}\left(\mathbf{D}_{i j}^{\alpha_{q}}+\mathbf{D}_{i j}^{1+\alpha_{q}}\right)\right] u_{N}\left(\xi_{j}\right)=f\left(\xi_{i}\right), \\
& \sum_{j=2}^{N} \mathscr{D}_{i, j} u_{N}\left(\xi_{j}\right)=f\left(\xi_{i}\right), \quad i=2,3, \cdots, N .
\end{aligned}
$$

REMARK 4.1. Multi-term problems can be generalized to the distributed order counterparts through the definition of distribution function $\phi(\alpha)$. For instance, if the operator consists 
of multiple fractional orders $0<\alpha_{1}<\alpha_{2}<\cdots<\alpha_{P} \leq 2$, the corresponding multi-term problem

$$
\sum_{p=1}^{p=P}{ }_{0} \mathcal{D}_{t}^{\alpha_{p}} u(t)=f(t)
$$

can be represented as a distributed order problem of the form 2.25), in which $\phi(\alpha)=$ $\sum_{p=1}^{p=P} \delta\left(\alpha-\alpha_{p}\right)$. We note that in this case, the distributed fractional Sobolev space, ${ }^{\phi} \mathcal{H}(\mathbb{R})$, coincides with the fractional Sobolev space, $H^{\alpha_{P}}(\mathbb{R})$. The choice of collocation/interpolation points is the key to construct well-conditioned linear systems with optimal approximability. In the present work, we leave $\mu$ in expansion (4.1) as a free interpolation parameter to capture possible singularities and employ the zeros of Legendre polynomials as the interpolation collocation/interpolation points.

5. Numerical Simulations. In order to examine the convergence of the schemes with modal and nodal expansions, we consider problems with smooth and non-smooth solutions.

5.1. Smooth Solutions. Let $\alpha \in[0,2]$ and consider the following two cases:

- Case I: $u^{e x t}=t^{5}, \phi(\alpha)=\Gamma(6-\alpha) / 5 !, f(t)=\frac{\left(t^{5}-t^{3}\right)}{\log (t)}$

- Case II: $u^{e x t}=t^{3}, \phi(\alpha)=\Gamma(4-\alpha) \sinh (\alpha), f(t)=\frac{6 t\left(t^{2}-\cosh (2)-\sinh (2) \log (t)\right)}{\left(\log (t)^{2}-1\right)}$.

By taking the simulation time $T=2$ and for different choices of $\mu$, we provide the convergence study in $L^{\infty}$-norm, $L^{2}$-norm, $H^{\mu_{1}}$-norm and ${ }^{\phi} \mathcal{H}$-norm using the PG scheme and in $L^{\infty}$-norm using the collocation scheme. It is observed that the choice of $\mu$ has an important effect on the convergence behaviour of the scheme. For instance, since the exact solution is a polynomial, as $\mu \rightarrow 1$, we recover the exponential convergence in capturing the exact solution.

Table 5.1: Case-I; PG scheme convergence study in $L^{\infty}$-norm, $L^{2}$-norm, $H^{\mu_{1}}$-norm and ${ }^{\phi} \mathcal{H}$ norm, where $T=2$.

\begin{tabular}{|c|c|c|c|c|}
\hline $\mathrm{N}$ & $\overline{L^{\infty} \text {-Error }}$ & $\overline{L^{2} \text {-Error }}$ & $\overline{H^{\mu_{1}} \text {-Error }}$ & $\overline{{ }^{\phi} \mathcal{H} \text {-Error }}$ \\
\hline 2 & 9.49784 & 3.38063 & 20.604 & 6.52507 \\
\hline 4 & 0.163486 & 0.0823368 & 0.802757 & 0.187176 \\
\hline 6 & $9.71043 \times 10^{-8}$ & $6.7433 \times 10^{-8}$ & $8.37613 \times 10^{-7}$ & $1.70551 \times 10^{-1}$ \\
\hline 8 & $2.9053 \times 10^{-9}$ & $2.32457 \times 10^{-9}$ & $3.53574 \times 10^{-8}$ & $6.59486 \times 10^{-9}$ \\
\hline \multirow[t]{2}{*}{10} & $2.27748 \times 10^{-10}$ & $2.01002 \times 10^{-10}$ & $3.67074 \times 10^{-9}$ & $6.38469 \times 10^{-10}$ \\
\hline & & $\mu_{1}=1.1$ & & \\
\hline$\overline{\mathrm{N}}$ & $\overline{L^{\infty} \text {-Error }}$ & $\overline{L^{2} \text {-Error }}$ & 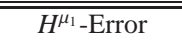 & 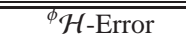 \\
\hline 2 & 9.6776 & 3.2898 & 23.3004 & 6.38693 \\
\hline 4 & 0.160434 & 0.0661304 & 0.872809 & 0.157957 \\
\hline 6 & 0.0000947942 & 0.0000589784 & 0.00107458 & 0.00015822 \\
\hline 8 & $3.10668 \times 10^{-6}$ & $2.19939 \times 10^{-6}$ & 0.0000507737 & $6.59429 \times 10^{-6}$ \\
\hline \multirow[t]{2}{*}{10} & $2.48519 \times 10^{-7}$ & $1.9822 \times 10^{-7}$ & $5.5753 \times 10^{-6}$ & $6.61409 \times 10^{-7}$ \\
\hline & & $\mu_{1}=1.5$ & & \\
\hline $\mathrm{N}$ & $L^{\infty}$-Error & $L^{2}$-Error & $H^{\mu_{1}}$-Error & ${ }^{\phi} \mathcal{H}$-Error \\
\hline 2 & 9.8476 & 3.10681 & 35.8457 & 5.96161 \\
\hline 4 & 0.102534 & 0.0264974 & 0.949183 & 0.0718496 \\
\hline 6 & 0.000584995 & 0.00015106 & 0.0117235 & 0.000524729 \\
\hline 8 & 0.0000272655 & $7.37649 \times 10^{-6}$ & 0.000989158 & 0.0000306404 \\
\hline 10 & $2.75271 \times 10^{-6}$ & $7.75346 \times 10^{-7}$ & 0.000158823 & $3.72512 \times 10^{-6}$ \\
\hline
\end{tabular}


Table 5.2: Case-II; PG scheme convergence study in $L^{\infty}$-norm, $L^{2}$-norm, $H^{\mu_{1}}$-norm and ${ }^{\phi} \mathcal{H}$-norm, where $T=2$.

\begin{tabular}{|c|c|c|c|c|}
\hline $\mathrm{N}$ & $L^{\infty}$-Error & $L^{2}$-Error & $H^{\mu_{1}}$-Error & ${ }^{\phi} \mathcal{H}$-Error \\
\hline 2 & 0.379134 & 0.325253 & 1.44392 & 1.86897 \\
\hline 4 & $6.80222 \times 10^{-7}$ & $6.33141 \times 10^{-7}$ & $4.61395 \times 10^{-6}$ & $5.29606 \times 10^{-6}$ \\
\hline 6 & $5.22608 \times 10^{-8}$ & $4.52071 \times 10^{-8}$ & $4.80236 \times 10^{-7}$ & $5.08899 \times 10^{-1}$ \\
\hline 8 & $1.27547 \times 10^{-8}$ & $9.98313 \times 10^{-9}$ & $1.0532 \times 10^{-7}$ & $1.049 \times 10^{-7}$ \\
\hline 10 & $7.31142 \times 10^{-9}$ & $7.21402 \times 10^{-9}$ & $3.44882 \times 10^{-8}$ & $3.39574 \times 10^{-8}$ \\
\hline \multicolumn{5}{|c|}{$\mu_{1}=1.1$} \\
\hline $\mathrm{N}$ & $L^{\infty}$-Error & $L^{2}$-Error & $H^{\mu_{1}}$-Error & ${ }^{\phi} \mathcal{H}$-Error \\
\hline 2 & 0.369682 & 0.263829 & 1.45384 & 1.62458 \\
\hline 4 & 0.000646557 & 0.000569995 & 0.00548608 & 0.00499413 \\
\hline 6 & 0.0000458334 & 0.0000438926 & 0.000636023 & 0.000511403 \\
\hline 8 & $7.74333 \times 10^{-6}$ & $7.36329 \times 10^{-6}$ & 0.000147177 & 0.000107932 \\
\hline 10 & $2.02013 \times 10^{-6}$ & $1.84714 \times 10^{-6}$ & 0.000048212 & 0.0000327428 \\
\hline \multicolumn{5}{|c|}{$\mu_{1}=1.5$} \\
\hline $\mathrm{N}$ & $L^{\infty}$-Error & $L^{2}$-Error & $H^{\mu_{1}}$-Error & ${ }^{\phi} \mathcal{H}$-Error \\
\hline 2 & 0.288508 & 0.114871 & 1.25471 & 0.848595 \\
\hline 4 & 0.00403916 & 0.00163979 & 0.0511667 & 0.0190804 \\
\hline 6 & 0.000406095 & 0.000169817 & 0.0106909 & 0.00268063 \\
\hline 8 & 0.0000789352 & 0.0000336939 & 0.00358698 & 0.000671243 \\
\hline 10 & 0.0000219275 & $9.49574 \times 10^{-6}$ & 0.00153771 & 0.000228446 \\
\hline
\end{tabular}

Table 5.3: Case-I and II; collocation scheme convergence study in $L^{\infty}$-norm, where $T=2$.

\begin{tabular}{ccccccc}
\hline \multicolumn{2}{c}{$\mu=1-10^{-10}$} & \multicolumn{2}{c}{$\mu=7 / 10$} & \multicolumn{2}{c}{$\mu=1 / 10$} \\
\hline $\mathrm{N}$ & Case-I & Case-II & Case-I & Case-II & Case-I & Case-II \\
\hline \hline 2 & $2.59 \times 10^{+1}$ & 5.74 & $3.0 \times 10^{+1}$ & 8.84 & $4.3 \times 10^{+1}$ & 19.91 \\
\hline 4 & $6.81 \times 10^{-1}$ & $5.30 \times 10^{-12}$ & $1.10 \times 10^{+1}$ & $2.58 \times 10^{-1}$ & $2.51 \times 10^{+1}$ & $1.01 \times 10^{-1}$ \\
\hline 6 & $3.87 \times 10^{-13}$ & $2.15 \times 10^{-13}$ & $1.43 \times 10^{-3}$ & $1.52 \times 10^{-3}$ & $3.48 \times 10^{-3}$ & $8.03 \times 10^{-3}$ \\
\hline 8 & $1.10 \times 10^{-14}$ & $2.68 \times 10^{-14}$ & $3.10 \times 10^{-5}$ & $3.34 \times 10^{-4}$ & $8.38 \times 10^{-5}$ & $1.83 \times 10^{-3}$ \\
\hline 10 & $8.75 \times 10^{-15}$ & $7.01 \times 10^{-15}$ & $2.12 \times 10^{-6}$ & $1.12 \times 10^{-4}$ & $1.0 \times 10^{-5}$ & $6.25 \times 10^{-4}$ \\
\hline
\end{tabular}

Tables 5.1 and 5.2 show the convergence behaviour of the simulation results based on the PG scheme for the two case-I and II respectively. Tables 5.3 shows the convergence behaviour of the simulation results based on the collocation scheme for the two cases I and II. Table 5.4 shows the convergence behaviour of the simulation results based on the collocation scheme for the case where the exact solution is the same as case-I but the distribution function is $\phi(\alpha)=\sum_{p=1}^{4} \delta\left(\alpha-\alpha_{p}\right)$ with the fractional orders $\{1 / 10,1 / 2,13 / 10,19 / 10\}$ and the forcing function is $f(t)=\sum_{p=1}^{4} \frac{120}{\Gamma\left(6-\alpha_{p}\right)} t^{5-\alpha_{p}}$.

Table 5.4: Multi-term case; collocation scheme convergence study in $L^{\infty}$-norm, where $T=2$.

\begin{tabular}{cccc}
\hline $\mathrm{N}$ & $\mu=1-10^{-10}$ & $\mu=7 / 10$ & $\mu=1 / 10$ \\
\hline \hline 6 & $2.99596 \times 10^{-4}$ & $1.93088 \times 10^{3}$ & $7.10859 \times 10^{-2}$ \\
\hline 10 & $4.40056 \times 10^{-7}$ & $7.90916 \times 10^{-6}$ & $1.95735 \times 10^{-4}$ \\
\hline 14 & $9.35031 \times 10^{-9}$ & $3.39228 \times 10^{-7}$ & $7.99603 \times 10^{-6}$ \\
\hline 18 & $9.15918 \times 10^{-10}$ & $3.53369 \times 10^{-8}$ & $8.27226 \times 10^{-7}$ \\
\hline
\end{tabular}

5.2. Non-Smooth Solutions. Since the exact solution is not always known and in contrast to the standard fractional ODEs where the forcing term gives some regularity information about the exact solution, in distributed order problems such a prediction is rather difficult to 
make. Hence, the fractional parameter $\mu$ can play the role of a fine-tuning knob giving the possibility of searching for the best/optimal case, where the highest rate can be achieved with minimal degrees of freedom. Here, we let $\alpha \in[0,1]$ and consider the following two cases of singular solution, where by the proper choice of $\mu$ we can easily capture the singularity of the solution.

- Case III: $u^{e x t}=t^{\mu_{0}}, \phi(\alpha)=\Gamma\left(1+\mu_{0}-\alpha\right) /\left(\mu_{0}\right) !, \mu_{0}=1 / 10,9 / 10$,

- Case IV: $u^{e x t}=t^{\mu_{0}} \sin (t), \phi(\alpha), \mu_{0}=75 / 100,25 / 100$.

In case-III, we are able to obtain the exact solution only with one term by choosing $\mu=\mu_{0}$. In case-IV, we take $\mu=\mu_{0}$ and expand $\sin (t)$ using Taylor series. Table 5.5 shows the $L^{2}$-norm convergence of the PG scheme for two different distribution functions.

Table 5.5: Case-IV; PG scheme convergence study in $L^{2}$-norm, where $T=2$.

\begin{tabular}{lcccc}
\hline \multicolumn{2}{c}{$\mu=75 / 100$} & \multicolumn{2}{c}{$\mu=25 / 100$} \\
\hline $\mathrm{N}$ & $\phi(\alpha)=1$ & $\phi(\alpha)=$ Normal & $\phi(\alpha)=1$ & $\phi(\alpha)=$ Normal \\
\hline \hline 2 & $1.56682 \times 10^{-1}$ & $1.62765 \times 10^{-1}$ & $1.5773 \times 10^{-1}$ & $1.548 \times 10^{-1}$ \\
\hline 4 & $3.13043 \times 10^{-3}$ & $3.3898 \times 10^{-3}$ & $3.4228 \times 10^{-3}$ & $3.28626 \times 10^{-3}$ \\
\hline 6 & $2.55359 \times 10^{-5}$ & $2.81522 \times 10^{-5}$ & $2.8956 \times 10^{-5}$ & $2.76729 \times 10^{-5}$ \\
\hline 8 & $1.13562 \times 10^{-1}$ & $1.2512 \times 10^{-1}$ & $4.24126 \times 10^{-1}$ & $1.40114 \times 10^{-1}$ \\
\hline 10 & $2.60471 \times 10^{-9}$ & $7.84647 \times 10^{-10}$ & $3.9524 \times 10^{-1}$ & $5.49882 \times 10^{-8}$ \\
\hline
\end{tabular}

5.3. Condition Number. The condition number of the constructed linear system is obtained for different distribution functions, $\phi(\alpha)$. Tables 5.6 and 5.7 show, respectively, the condition number of the constructed linear system for case-I and II based on PG and collocation scheme for the aforementioned distribution functions.

Table 5.6: Case-I and II; PG scheme condition number of the constructed linear system, where $T=2$.

\begin{tabular}{ccccccccc}
\hline & \multicolumn{2}{c}{$\mu=2-10^{-8}$} & \multicolumn{2}{c}{$\mu=2-10^{-1}$} & \multicolumn{2}{c}{$\mu=1+1 / 2$} & \multicolumn{2}{c}{$\mu=1+1 / 10$} \\
\hline $\mathrm{N}$ & Case-I & Case-II & Case-I & Case-II & Case-I & Case-II & Case-I & Case-II \\
\hline \hline 6 & 29706.682 & 4863.50 & 14319.465 & 2168.87 & 661.70145 & 70.1081 & 51.928935 & 7.19267 \\
\hline 10 & 240000.55 & 33494.8 & 90197.388 & 11817.9 & 1339.2855 & 130.925 & 42.754111 & 9.35597 \\
\hline 14 & 882010.62 & 118283 & 279501.78 & 35395.6 & 1941.9838 & 190.309 & 47.335770 & 13.0337 \\
\hline 18 & $2.2811229 \times 10^{6}$ & 301479 & 633307.06 & 79324.0 & 2505.5107 & 247.627 & 50.713428 & 16.7944 \\
\hline
\end{tabular}

Table 5.7: Case-I and II; collocation scheme condition number of the constructed linear system, where $T=2$.

\begin{tabular}{lcccccccc}
\hline & \multicolumn{2}{c}{$\mu=1-10^{-8}$} & \multicolumn{2}{c}{$\mu=1-10^{-1}$} & \multicolumn{2}{c}{$\mu=1 / 2$} & \multicolumn{2}{c}{$\mu=1 / 10$} \\
\hline $\mathrm{N}$ & Case-I & Case-II & Case-I & Case-II & Case-I & Case-II & Case-I & Case-II \\
\hline \hline 6 & 67.5606 & 345.045 & 60.3467 & 302.74 & 43.6649 & 191.058 & 36.0056 & 340.539 \\
\hline 10 & 386.339 & 2781.51 & 325.037 & 2309.25 & 214.935 & 1515.11 & 202.826 & 3554.95 \\
\hline 14 & 1330.11 & 10646.1 & 1076.14 & 8518.56 & 685.166 & 5435.91 & 713.002 & 16539.5 \\
\hline 18 & 3388.95 & 28619.5 & 2665.32 & 22290.4 & 1661.16 & 13964.9 & 3397.2 & 50911.1 \\
\hline
\end{tabular}

Moreover, three cases for the distribution function are considered: 1) the distribution is more biased toward the left of domain, 2) the distribution is symmetric and 3) the distribution is more biased toward the right of domain, namely left biased, symmetric and right biased respectively. The distribution functions are well-known normal, exponential, log-normal, Cauchy, Laplace, Beta and Maxwell distributions, however, they are truncated and normalized, see Fig 5.1 For these distributions, the condition number of the constructed linear system based on the two methods is computed and provided in Tables 5.8, 5.9 and 5.10, 


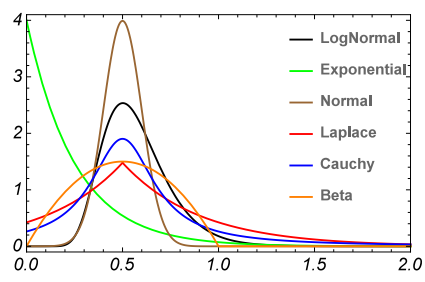

(a)

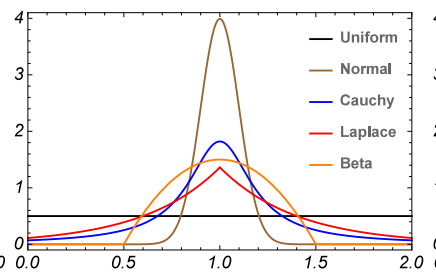

(b)

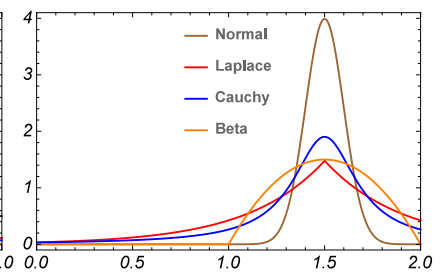

(c)

Fig. 5.1: Distribution functions: (a) Left biased (b) Symmetric (c) Right biased

Table 5.8: Left biased distribution function; PG (top) and collocation (bottom) scheme condition number of the constructed linear system, where $T=2$.

\begin{tabular}{|c|c|c|c|c|c|c|}
\hline $\mathbf{N}$ & LogNormal & Exponential & Normal & Laplace & Cauchy & Beta \\
\hline \multicolumn{7}{|c|}{$\mu=2-1 / 10$} \\
\hline 6 & 62101.5 & 130227 & 85410.4 & 21714.7 & 31361 & 70458.4 \\
\hline 10 & $1.28119 \times 10^{6}$ & $2.62266 \times 10^{6}$ & $2.15167 \times 10^{6}$ & 186527 & 294630 & $1.51681 \times 10^{6}$ \\
\hline 14 & $9.84911 \times 10^{6}$ & $1.61563 \times 10^{7}$ & $1.98724 \times 10^{7}$ & 668178 & $1.04066 \times 10^{6}$ & $1.22216 \times 10^{6}$ \\
\hline 18 & $4.48721 \times 10^{7}$ & $5.34428 \times 10^{7}$ & $1.0748 \times 10^{8}$ & $1.62018 \times 10^{6}$ & $2.41399 \times 10^{6}$ & $5.86944 \times 10^{6}$ \\
\hline \multicolumn{7}{|c|}{$\mu=1+1 / 10$} \\
\hline 6 & 200.626 & 505.679 & 300.643 & 71.5348 & 100.504 & 233.849 \\
\hline 10 & 654.259 & 1397.74 & 1309.12 & 91.8644 & 140.467 & 816.254 \\
\hline 14 & 1322.63 & 1969.15 & 3437.72 & 64.4093 & 98.4193 & 1780.96 \\
\hline 18 & 2145.61 & 2178 & 7037.99 & 70.7541 & 91.8767 & 3134.64 \\
\hline $\mathbf{N}$ & LogNormal & Exponential & Normal & Laplace & Cauchy & Beta \\
\hline \multicolumn{7}{|c|}{$\mu=1-1 / 10$} \\
\hline 6 & 20.1001 & 9.42989 & 13.3574 & 51.9103 & 39.5064 & 16.8765 \\
\hline 10 & 48.3364 & 25.6229 & 26.2852 & 237.89 & 169.114 & 38.3283 \\
\hline 14 & 91.0866 & 55.5712 & 46.5097 & 714.563 & 503.229 & 65.4666 \\
\hline 18 & 143.171 & 126.19 & 73.3388 & 1672.61 & 1185.15 & 97.5852 \\
\hline \multicolumn{7}{|c|}{ 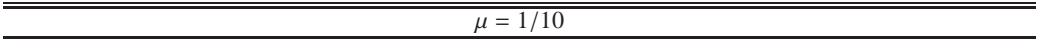 } \\
\hline 6 & 15.7319 & 5.67323 & 11.4277 & 33.4341 & 26.3924 & 13.3573 \\
\hline 10 & 40.2359 & 18.0958 & 22.8619 & 150.375 & 102.285 & 30.7949 \\
\hline 14 & 71.0054 & 37.1664 & 35.1324 & 435.867 & 309.089 & 52.9303 \\
\hline 18 & 110.725 & 80.9506 & 48.0051 & 1032.88 & 742.025 & 81.7202 \\
\hline
\end{tabular}

6. Summary and Discussion. We developed two spectrally-accurate schemes, namely the Petrov-Galerkin spectral method and the fractional spectral collocation method for distributed order fractional differential equations. The two schemes were constructed based on the recently developed spectral theory for fractional Sturm-Liouville problems (FSLPs). In the Petrov-Galerkin method, we employed the Jacobi poly-fractonomials as the bases, which are the eigenfunctions of FSLP-I, and the poly-fractonomial eigenfunctions of FSLP-II as the test functions. We carried out the discrete stability analysis of the proposed scheme employing some equivalent/bilinear-induced norms based on the defined distributed Sobolev spaces and their associated norms. In addition, we performed a convergence study of the proposed scheme. In the collocation method, we employed fractional Lagrange interpolants satisfying the Kronecker delta property at the collocation points, and then we obtained the corresponding distributed differentiation matrices to discretize the strong problem.

The existing schemes in the literature are mostly employing finite difference methods. The main challenge in these methods, in comparison to spectral methods, is the history calculation as well as extensive memory allocation while they deliver fixed algebraic accuracies. 
Table 5.9: Symmetric distribution function; PG (left) and collocation (right) scheme condition number of the constructed linear system, where $T=2$.

\begin{tabular}{lccccc}
\hline $\mathbf{N}$ & Uniform & Normal & Cauchy & Laplace & Beta \\
\hline \hline \multicolumn{5}{c}{$\mu=2-1 / 10$} \\
\hline 6 & 3104.6331 & 16261.6 & 9328.53 & 6969.22 & 13404.7 \\
\hline 10 & 17244.219 & 220205 & 80110.2 & 51304.3 & 157767 \\
\hline 14 & 52095.460 & $1.28827 \times 10^{6}$ & 308549 & 179737 & 817268 \\
\hline 18 & 117338.89 & $4.86517 \times 10^{6}$ & 803362 & 441628 & $2.77821 \times 10^{6}$ \\
\hline \hline \multicolumn{5}{c}{$\mu=1+1 / 10$} \\
\hline 6 & 9.5451677 & 44.0857 & 27.6858 & 17.9872 & 37.4224 \\
\hline 10 & 11.211269 & 85.3822 & 30.0092 & 21.361 & 64.2331 \\
\hline 14 & 12.360897 & 127.844 & 30.9343 & 20.1997 & 87.2794 \\
\hline 18 & 15.900925 & 172.888 & 34.2126 & 22.1982 & 107.403 \\
\hline \multicolumn{7}{c}{}
\end{tabular}

\begin{tabular}{lccccc}
\hline $\mathbf{N}$ & Uniform & Normal & Cauchy & Laplace & Beta \\
\hline \hline \multicolumn{5}{c}{$\mu=1-1 / 10$} \\
\hline 6 & 219.733 & 81.9543 & 118.433 & 140.922 & 95.0983 \\
\hline 10 & 1592.05 & 284.171 & 598.247 & 798.174 & 375.673 \\
\hline 14 & 5769.24 & 620.056 & 1788.85 & 2549.17 & 905.972 \\
\hline 18 & 14944.9 & 1097.37 & 4122.7 & 6115.65 & 1737.18 \\
\hline \hline \multicolumn{5}{c}{$\mu=1 / 10$} \\
\hline 6 & 183.984 & 55.8908 & 72.0876 & 78.4252 & 62.069 \\
\hline 10 & 1854.46 & 198.226 & 360.943 & 506.994 & 248.186 \\
\hline 14 & 8678.37 & 439.316 & 1113.83 & 1595.84 & 589.611 \\
\hline 18 & 26873.1 & 786.655 & 2600.56 & 3954.72 & 1171.64 \\
\hline
\end{tabular}

Table 5.10: Right biased distribution function; PG (left) and collocation (right) scheme condition number of the constructed linear system, where $T=2$.

\begin{tabular}{lllll}
\hline $\mathbf{N}$ & Normal & Laplace & Cauchy & Beta \\
\hline \hline \multicolumn{5}{c}{$\mu=2-1 / 10$} \\
\hline 6 & 2985.08 & 2274.39 & 2368.82 & 2105.03 \\
\hline 10 & 23439.3 & 13948.9 & 15471.9 & 13474.7 \\
\hline 14 & 92925.7 & 45201.4 & 52626.9 & 45325.7 \\
\hline 18 & 259993 & 107082 & 129454 & 110813 \\
\hline \hline \multicolumn{5}{c}{$\mu=1+1 / 10$} \\
\hline 6 & 6.99801 & 6.60933 & 6.54308 & 6.18762 \\
\hline 10 & 10.8049 & 9.81523 & 10.0861 & 10.1067 \\
\hline 14 & 15.1563 & 14.2958 & 14.0265 & 14.6423 \\
\hline 18 & 19.4415 & 18.4003 & 18.8445 & 18.8791 \\
\hline
\end{tabular}

\begin{tabular}{lcccc}
\hline $\mathbf{N}$ & Normal & Laplace & Cauchy & Beta \\
\hline \hline \multicolumn{5}{c}{$\mu=1-1 / 10$} \\
\hline 6 & 329.01 & 328.433 & 333.499 & 360.053 \\
\hline 10 & 2022.09 & 2330.68 & 2275.43 & 2501.41 \\
\hline 14 & 6299.73 & 8170.64 & 7736.87 & 8579.48 \\
\hline 18 & 14429.7 & 20615.9 & 19067.6 & 21250 \\
\hline \hline \multicolumn{5}{c}{$\mu=1 / 10$} \\
\hline 6 & 278.525 & 333.384 & 322.187 & 378.704 \\
\hline 10 & 1539.25 & 2647.09 & 2246.71 & 2727.03 \\
\hline 14 & 4701.38 & 10365.3 & 8041.12 & 9884.8 \\
\hline 18 & 10622.6 & 28764.7 & 20895.4 & 25800.8 \\
\hline
\end{tabular}

The recent spectral theory on fractional Sturm-Liouville problems (FSLPs) in [61] naturally motivates the use of Petrov-Galerkin spectral methods, where the arising bilinear forms are comprised of left- and right-sided fractional derivatives. The eigen-functions of FSLPs can be employed naturally as the bases and test spaces, where their left- and right-sided derivatives are obtained analytically. These functions consist of a polynomial part and a fractional part, where the former leaves the fractional order, $\mu$, as a free parameter to capture solution singularities, hence, to tune up the accuracy of the scheme from being algebraically convergent to exponential convergent. In fact, the Case-III of numerical examples demonstrated how a proper choice of fractional part of the bases provides the exact solution with only one term expansion. Furthermore, we proved that the distributed bilinear form can be approximated with a spectral/exponential accuracy using a proper quadrature rule. The PG spectral method treats the nonlocal effects efficiently through a global spectral method and provides a nice mathematical framework for performing theoretical studies, however, treating nonlinear problems remains a challenge. To this end, we constructed a spectrally accurate fractional spectral collocation method employing fractional Lagrange interpolants, where for linear problems the two developed schemes become equivalent in terms of the rate of convergence.

The distribution function, $\phi(\alpha)$, defined the distribution of the differentiation fractionalorder, $\alpha$, and it could arbitrarily confine the domain over which the fractional differentiation is taken. If $\phi$ was integrable in a compact support in $\left[\alpha_{\min }, \alpha_{\max }\right]$, then $H^{\alpha_{\min }}(\mathbb{R}) \supseteq{ }^{\phi} \mathcal{H}(\mathbb{R}) \supseteq$ $H^{\alpha_{\max }}(\mathbb{R})$. Hence, $\phi$ could play a crucial rule in defining the underlying solution space properly. In anomalous physical processes, the distribution function can be obtained from experimental data, where the inherent data uncertainty can be incorporated through the $\phi$ obtained from the observed data, hence, leading to a robust data-driven simulation framework for multi-physics problems.

\section{Appendix A. Proof Of Lemma 2.1].}


Proof. Following [1] and for $\sigma>0, \alpha>-1, \beta>-1$, and $\forall x \in[-1,1]$ we have

$$
(1+x)^{\beta+\sigma} \frac{P_{n}^{\alpha-\sigma, \beta+\sigma}(x)}{P_{n}^{\alpha-\sigma, \beta+\sigma}(-1)}=\frac{\Gamma(\beta+\sigma+1)}{\Gamma(\beta+1) \Gamma(\sigma) P_{n}^{\alpha, \beta}(-1)} \int_{-1}^{x} \frac{(1+s)^{\beta} P_{n}^{\alpha, \beta}(s)}{(x-s)^{1-\sigma}} d s,
$$

and

$$
(1-x)^{\alpha+\sigma} \frac{P_{n}^{\alpha+\sigma, \beta-\sigma}(x)}{P_{n}^{\alpha+\sigma, \beta-\sigma}(+1)}=\frac{\Gamma(\alpha+\sigma+1)}{\Gamma(\alpha+1) \Gamma(\sigma) P_{n}^{\alpha, \beta}(+1)} \int_{x}^{1} \frac{(1-s)^{\alpha} P_{n}^{\alpha, \beta}(s)}{(s-x)^{1-\sigma}} d s .
$$

By the definition of the left-sided Riemann-Liouville integral ${ }_{-1}^{R L} \mathcal{I}_{x}^{\sigma}$ and evaluating the special end-values $P_{n}^{\alpha-\sigma, \beta+\sigma}(-1)$ and $P_{n}^{\alpha, \beta}(-1)$, we can re-write $\mathrm{A} .1$ as

$$
{ }_{-1}^{R L} I_{x}^{\sigma}\left\{(1+x)^{\beta} P_{n}^{\alpha, \beta}(x)\right\}=\frac{\Gamma(n+\beta+1)}{\Gamma(n+\beta+\sigma+1)}(1+x)^{\beta+\sigma} P_{n}^{\alpha-\sigma, \beta+\sigma}(x),
$$

where, by taking the fractional derivative ${ }_{-1}^{R L} \mathcal{D}_{x}^{\sigma}$ on the both sides, we obtain

$$
{ }_{-1}^{R L} \mathcal{D}_{x}^{\sigma}\left\{(1+x)^{\beta+\sigma} P_{n}^{\alpha-\sigma, \beta+\sigma}(x)\right\}=\frac{\Gamma(n+\beta+\sigma+1)}{\Gamma(n+\beta+1)}(1+x)^{\beta} P_{n}^{\alpha, \beta}(x) .
$$

Hence, taking $\beta+\sigma=\mu, \alpha-\sigma=-\mu$ in $\widehat{\text { A.3 }}$, and shifting from $n$ to $n-1$, we obtain

$$
\begin{aligned}
{ }_{-1}^{R L} \mathcal{D}_{x}^{\sigma}\left\{{ }^{(1)} \mathcal{P}_{n}^{\mu}(x)\right\} & =\frac{\Gamma(n+\mu)}{\Gamma(n+\mu-\sigma)}(1+x)^{\mu-\sigma} P_{n-1}^{\sigma-\mu, \mu-\sigma}(x), \\
& =\frac{\Gamma(n+\mu)}{\Gamma(n+\mu-\sigma)}(1+x)^{\eta} P_{n-1}^{-\eta, \eta}(x), \\
& =\frac{\Gamma(n+\mu)}{\Gamma(n+\mu-\sigma)}{ }^{(1)} \mathcal{P}_{n}^{\eta}(x),
\end{aligned}
$$

where $\eta=\mu-\sigma$. Moreover, by the definition of the right-sided Riemann-Liouville integral ${ }_{x}^{R L} \mathcal{I}_{1}^{\sigma}$ and evaluating the special end-values $P_{n}^{\alpha-\sigma, \beta+\sigma}(+1)$ and $P_{n}^{\alpha, \beta}(+1)$, we can re-write A.2 as

$$
{ }_{x}^{R L} \mathcal{I}_{1}^{\sigma}\left\{(1-x)^{\alpha} P_{n}^{\alpha, \beta}(x)\right\}=\frac{\Gamma(n+\alpha+1)}{\Gamma(n+\alpha+\sigma+1)}(1-x)^{\alpha+\sigma} P_{n}^{\alpha+\sigma, \beta-\sigma}(x) .
$$

In a similar fashion, by taking the fractional derivative ${ }_{x}^{R L} \mathcal{D}_{-1}^{\sigma}$ on the both sides, we obtain

$$
{ }_{x}^{R L} \mathcal{D}_{1}^{\sigma}\left\{(1-x)^{\alpha+\sigma} P_{n}^{\alpha+\sigma, \beta-\sigma}(x)\right\}=\frac{\Gamma(n+\alpha+\sigma+1)}{\Gamma(n+\alpha+1)}(1-x)^{\alpha} P_{n}^{\alpha, \beta}(x) .
$$

Next, by taking $\alpha+\sigma=\mu, \beta-\sigma=-\mu$ in $\underline{\mathrm{A} .5}$, and again shifting from $n$ to $n-1$ we have

$$
\begin{aligned}
{ }_{x}^{R L} \mathcal{D}_{1}^{\sigma}\left\{{ }^{(2)} \mathcal{P}_{n}^{\mu}(x)\right\} & =\frac{\Gamma(n+\mu)}{\Gamma(n+\mu-\sigma)}(1-x)^{\mu-\sigma} P_{n-1}^{\mu-\sigma, \sigma-\mu}(x) . \\
& =\frac{\Gamma(n+\mu)}{\Gamma(n+\mu-\sigma)}(1-x)^{\eta} P_{n-1}^{\eta,-\eta}(x), \\
& =\frac{\Gamma(n+\mu)}{\Gamma(n+\mu-\sigma)}{ }^{(2)} \mathcal{P}_{n}^{\eta}(x),
\end{aligned}
$$

and that completes the proof. $\square$

\section{Appendix B. Proof Of Theorem 2.1.}


Proof. Let $\phi$ be bounded in $\left(\alpha_{\min }, \alpha_{\max }\right)$. Then,

(B.1)

$$
C_{1} \phi_{\min } A \leq\|\cdot\|_{l, \phi, I}^{2} \leq C_{2} \phi_{\max } A,
$$

$$
C_{3} \phi_{\min } B \leq\|\cdot\|_{r, \phi, I}^{2} \leq C_{4} \phi_{\max } B
$$

where

$$
\begin{aligned}
& A=\|\cdot\|_{L^{2}(I)}^{2}+\int_{\alpha_{\min }}^{\alpha_{\max }}\left\|{ }_{x_{L}}^{R L} \mathcal{D}_{x}^{\alpha}(\cdot)\right\|_{L^{2}(I)}^{2} d \alpha, \\
& B=\|\cdot\|_{L^{2}(I)}^{2}+\int_{\alpha_{\min }}^{\alpha_{\max }}\left\|{ }_{x}^{R L} \mathcal{D}_{x_{R}}^{\alpha}(\cdot)\right\|_{L^{2}(I)}^{2} d \alpha,
\end{aligned}
$$

and $C_{1}, C_{2}, C_{3}$, and $C_{4}$ are positive constants. From [35], we know that $\forall \alpha=s$ fixed, $\|\cdot\|_{l, s, I} \sim\|\cdot\|_{r, s, I}$ that is $\left\|{ }_{x_{L}}^{R L} \mathcal{D}_{x}^{s}(\cdot)\right\|_{L^{2}(I)}^{2} \sim\left\|{ }_{x}{ }_{x} \mathcal{D}_{x_{R}}^{s}(\cdot)\right\|_{L^{2}(I)}^{2}$, hence let $\vec{s}=\left\{s_{1}, s_{2}, \cdots, s_{Q}\right\}$, and similarly $\forall s=s_{q}$ the aforementioned equivalence holds. Therefore, any linear combination of

$$
\sum_{q=1}^{Q} w_{q}\left\|{ }_{x_{L}}^{R L} \mathcal{D}_{x}^{s}(\cdot)\right\|_{L^{2}(I)}^{2} \sim \sum_{q=1}^{Q} w_{q}\left\|{ }_{x}^{R L} \mathcal{D}_{x_{R}}^{s}(\cdot)\right\|_{L^{2}(I)}^{2} .
$$

Taking $Q \longrightarrow \infty$ and assuming $\left\{w_{q}, s_{q}\right\}$ to be Riemann integral weights and points in $\left[\alpha_{\min }, \alpha_{\max }\right]$,

$$
\int_{\alpha_{\min }}^{\alpha_{\max }}\left\|{ }_{x_{L}}^{R L} \mathcal{D}_{x}^{s}(\cdot)\right\|_{L^{2}(I)}^{2} \sim \int_{\alpha_{\min }}^{\alpha_{\max }}\left\|{ }_{x}^{R L} \mathcal{D}_{x_{R}}^{s}(\cdot)\right\|_{L^{2}(I)}^{2} .
$$

By adding $\|\cdot\|_{L^{2}(I)}^{2}$ to the both sides of the above equivalence, we obtain $A \sim B$; and by (B.1), $\|\cdot\|_{l, \phi, I} \sim\|\cdot\|_{r, \phi, I}$.

In addition, from [35], we know that $\forall \alpha=s$ fixed, $\|\cdot\|_{s, I}^{2} \sim\|\cdot\|_{l, s, I}^{2}$. Let $\vec{s}=\left\{s_{1}, s_{2}, \cdots, s_{Q}\right\}$ thus $\forall s=s_{q} \in \vec{s},\|\cdot\|_{s_{q}, I}^{2} \sim\|\cdot\|_{l, s_{q}, I}^{2}$. Therefore, for any linear combination of $\sum_{q=1}^{Q} w_{q}\|\cdot\|_{s_{q}, I}^{2} \sim$ $\sum_{q=1}^{Q} w_{q}\|\cdot\|_{l, s_{q}, I}^{2}$. Taking $Q \longrightarrow \infty$, we obtain:

$$
\int_{\alpha_{\min }}^{\alpha_{\max }}\|\cdot\|_{\alpha, I}^{2} d \alpha \sim \int_{\alpha_{\min }}^{\alpha_{\max }}\|\cdot\|_{l, \alpha, I}^{2} d \alpha
$$

where the right hand side of the equivalence is

$$
\begin{aligned}
\int_{\alpha_{\min }}^{\alpha_{\max }}\left(\|\cdot\|_{L^{2}(I)}^{2}+\left\|{ }_{x_{L}}^{R L} \mathcal{D}_{x}^{\alpha}(\cdot)\right\|_{L^{2}(I)}^{2}\right) d \alpha & =\left(\alpha_{\max }-\alpha_{\min }\right)\|\cdot\|_{L^{2}(I)}^{2}+\int_{\alpha_{\min }}^{\alpha_{\max }}\left\|{ }_{x_{L}}^{R L} \mathcal{D}_{x}^{\alpha}(\cdot)\right\|_{L^{2}(I)}^{2} d \alpha, \\
& \sim\|\cdot\|_{l, \phi, I}^{2} \sim\|\cdot\|_{r, \phi, I}^{2}
\end{aligned}
$$

Therefore,

$$
\int_{\alpha_{\min }}^{\alpha_{\max }}\|\cdot\|_{\alpha, I}^{2} d \alpha \sim\|\cdot\|_{l, \phi, I}^{2} \sim\|\cdot\|_{r, \phi, I}^{2}
$$

We can also show that

$$
\phi_{\min } \int_{\alpha_{\min }}^{\alpha_{\max }}\left\|\left(1+|\omega|^{2}\right)^{\frac{\alpha}{2}} \mathcal{F}(\cdot)(\omega)\right\|_{L^{2}(\mathbb{R})}^{2} d \alpha \leq\|\cdot\|_{\phi, \mathbb{R}}^{2} \leq \phi_{\max } \int_{\alpha_{\min }}^{\alpha_{\max }}\left\|\left(1+|\omega|^{2}\right)^{\frac{\alpha}{2}} \mathcal{F}(\cdot)(\omega)\right\|_{L^{2}(\mathbb{R})}^{2} d \alpha .
$$


Because of the non-negativity of the norms, we have

$$
\begin{aligned}
& \phi_{\min } \inf _{\tilde{v} \in \epsilon^{\phi} \mathcal{H}(\mathbb{R}),\left.\tilde{v}\right|_{I}=(\cdot)} \int_{\alpha_{\min }}^{\alpha_{\max }}\left\|\left(1+|\omega|^{2}\right)^{\frac{\alpha}{2}} \mathcal{F}(\tilde{v})(\omega)\right\|_{L^{2}(\mathbb{R})}^{2} d \alpha \\
& \leq \inf _{\tilde{v} \in \mathcal{H}_{\mathcal{H}(\mathbb{R}), \tilde{v} \tilde{v}_{l}=(\tilde{v})}}\|\cdot\|_{\phi, \mathbb{R}}^{2} \leq \\
& \phi_{\max } \inf _{\tilde{v} \in \mathcal{H}^{\phi} \mathcal{H}(\mathbb{R}),\left.\tilde{v}\right|_{l}=(\cdot)} \int_{\alpha_{\min }}^{\alpha_{\max }}\left\|\left(1+|\omega|^{2}\right)^{\frac{\alpha}{2}} \mathcal{F}(\tilde{v})(\omega)\right\|_{L^{2}(\mathbb{R})}^{2} d \alpha .
\end{aligned}
$$

In general, ${ }^{\phi} \mathcal{H}(\mathbb{R}) \subset H^{\alpha_{\max }}(\mathbb{R}), \forall \alpha \in\left[\alpha_{\min }, \alpha_{\max }\right]$. Therefore, we have:

$$
\begin{aligned}
& \inf _{\tilde{v} \in \mathcal{H}_{\mathcal{H}}(\mathbb{R}),\left.\tilde{v}\right|_{I}=(\cdot)} \int_{\alpha_{\min }}^{\alpha_{\max }}\left\|\left(1+|\omega|^{2}\right)^{\frac{\alpha}{2}} \mathcal{F}(\tilde{v})(\omega)\right\|_{L^{2}(\mathbb{R})}^{2} d \alpha, \\
= & \int_{\alpha_{\min }}^{\alpha_{\max }} \inf _{\tilde{v} \epsilon^{\phi} \mathcal{H}(\mathbb{R}),\left.\tilde{v}\right|_{I}=(\cdot)}\left\|\left(1+|\omega|^{2}\right)^{\frac{\alpha}{2}} \mathcal{F}(\tilde{v})(\omega)\right\|_{L^{2}(\mathbb{R})}^{2} d \alpha, \\
\leq & C \int_{\alpha_{\min }}^{\alpha_{\max }} \inf _{\tilde{v} \in H^{\alpha}(\mathbb{R}),\left.\tilde{v}\right|_{l}=(\cdot)}\left\|\left(1+|\omega|^{2}\right)^{\frac{\alpha}{2}} \mathcal{F}(\tilde{v})(\omega)\right\|_{L^{2}(\mathbb{R})}^{2} d \alpha, \\
= & C \int_{\alpha_{\min }}^{\alpha_{\max }}\|\cdot\|_{\alpha, I}^{2} d \alpha .
\end{aligned}
$$

However, for some choices of $\phi=\delta\left(\alpha-\alpha_{\min }\right)$ and thus ${ }^{\phi} \mathcal{H}(\mathbb{R}) \supset H^{\alpha_{\max }}(\mathbb{R}), \forall \alpha \in\left[\alpha_{\min }, \alpha_{\max }\right]$. Therefore,

$$
\begin{aligned}
& \int_{\alpha_{\min }}^{\alpha_{\max }} \inf _{\tilde{v} \epsilon^{\phi} \mathcal{H}(\mathbb{R}), \tilde{v} l_{I}=(\cdot)}\left\|\left(1+|\omega|^{2}\right)^{\frac{\alpha}{2}} \mathcal{F}(\tilde{v})(\omega)\right\|_{L^{2}(\mathbb{R})}^{2} d \alpha, \\
\geq & \widetilde{C} \int_{\alpha_{\min }}^{\alpha_{\max }} \inf _{\tilde{v} \in H^{\alpha}(\mathbb{R}), \tilde{v} \tilde{v}_{I}=(\cdot)}\left\|\left(1+|\omega|^{2}\right)^{\frac{\alpha}{2}} \mathcal{F}(\tilde{v})(\omega)\right\|_{L^{2}(\mathbb{R})}^{2} d \alpha, \\
= & \widetilde{C} \int_{\alpha_{\min }}^{\alpha_{\max }}\|\cdot\|_{\alpha, I}^{2} d \alpha,
\end{aligned}
$$

which by (B.3) and (2.14), we get

$$
\int_{\alpha_{\min }}^{\alpha_{\max }}\|\cdot\|_{\alpha, I}^{2} d \alpha \sim\|\cdot\|_{\phi, I}^{2}
$$

Comparing (B.2) and (B.4), we have

$$
\|\cdot\|_{\phi, I}^{2} \sim\|\cdot\|_{l, \phi, I}^{2} \sim\|\cdot\|_{r, \phi, I}^{2}
$$

REMARK B.1. We note that if $\phi=\delta(\alpha-s)$, we recover the standard ${ }_{x_{L}}^{R L} \mathcal{D}_{x}^{s}(u)=f$, where the equivalence between the corresponding $\|\cdot\|_{l, s, I},\|\cdot\|_{r, s, I}$, and $\|\cdot\|_{s, I}$ has been already established. Moreover, we note that for the case $\phi \in L^{1}\left(\left[\alpha_{\min }, \alpha_{\max }\right)\right.$ containing finitely many singularities at $\alpha_{1}, \alpha_{2}, \cdots, \alpha_{m}$, the whole interval $\left[\alpha_{\min }, \alpha_{\max }\right]$ and the integration can be written as

$$
\int_{\alpha_{\text {min }}}^{\alpha_{1}} \phi(\alpha){ }_{x_{L}}^{R L} \mathcal{D}_{x}^{S}(u) d \alpha+\int_{\alpha_{1}}^{\alpha_{2}} \phi(\alpha){ }_{x_{L}}^{R L} \mathcal{D}_{x}^{s}(u) d \alpha+\cdots+\int_{\alpha_{m}}^{\alpha_{\max }} \phi(\alpha){ }_{x_{L}}^{R L} \mathcal{D}_{x}^{s}(u) d \alpha,
$$

where all the previous steps in the proof can apply in each interval.

\section{Appendix C. Proof of Lemma 2.4).}


Proof. Since $u(a)=0$, by (2.5) ${ }_{a} \mathcal{D}_{x}^{2 \mu} u={ }_{a} \mathcal{D}_{x}^{\mu}\left({ }_{a} \mathcal{D}_{x}^{\mu} u\right)$. Taking $\widetilde{u}(x)={ }_{a} \mathcal{D}_{x}^{\mu} u$, we have

$$
\begin{aligned}
\left({ }_{a} \mathcal{D}_{x}^{2 \mu} u, v\right) & =\left({ }_{a} \mathcal{D}_{x}^{\mu} \widetilde{u}, v\right), \\
& =\frac{1}{\Gamma(1-\mu)} \int_{a}^{b}\left[\frac{d}{d x} \int_{a}^{x} \frac{\widetilde{u}(s) d s}{(x-s)^{\mu}}\right] v(x) d x, \\
& =\left\{\frac{v(x)}{\Gamma(1-\mu)} \int_{a}^{x} \frac{\widetilde{u}(s) d s}{(x-s)^{\mu}}\right\}_{x=a}^{x=b} \\
& -\frac{1}{\Gamma(1-\mu)} \int_{a}^{b}\left[\int_{a}^{x} \frac{\widetilde{u}(s) d s}{(x-s)^{\mu}}\right] \frac{d v(x)}{d x} d x, \quad \text { by } v(b)=0, \\
& =-v(a) \lim _{x \rightarrow a} I_{x}^{1-\mu} \widetilde{u}-\frac{1}{\Gamma(1-\mu)} \int_{a}^{b} \int_{s}^{b} \frac{\frac{d v(x)}{d x} d x}{(x-s)^{\mu}} \widetilde{u}(s) d s,
\end{aligned}
$$

which make sense when the interior term $\int_{s}^{b} \frac{\frac{d(x)}{d x} d x}{(x-s)^{\mu}}$ is integrable in $(a, b)$. Taking into account that $v(a)$ is bounded, we can show that the boundary term $v(a) \lim _{x \rightarrow a_{a}} I_{x}^{1-\mu} \widetilde{u}$ also vanishes as

$$
\begin{aligned}
\lim _{x \rightarrow a}\left|{ }_{a} I_{x}^{1-\mu} \widetilde{u}\right| & =\lim _{x \rightarrow a} \frac{1}{\Gamma(1-\mu)}\left|\int_{a}^{x} \frac{\widetilde{u}(s) d s}{(x-s)^{\mu}}\right|, \\
& \leq \lim _{x \rightarrow a} \frac{1}{\Gamma(1-\mu)}\left|\int_{a}^{x} \frac{d s}{(x-s)^{\mu}}\right|\|\widetilde{u}\|_{L^{\infty}}, \\
& =\lim _{x \rightarrow a} \frac{1}{\Gamma(1-\mu)} \frac{(x-a)^{1-\mu}}{1-\mu}\|\widetilde{u}\|_{L^{\infty}}=0 .
\end{aligned}
$$

Moreover, it is easy to check that

$$
\begin{aligned}
\frac{d}{d s} \int_{s}^{b} \frac{v(x) d x}{(x-s)^{\mu}} & =\frac{d}{d s}\left\{\left.\frac{v(x)(x-s)^{1-\mu}}{1-\mu}\right|_{x=s} ^{x=b}-\frac{1}{1-\mu} \int_{s}^{b} \frac{d v(x)}{d x}(x-s)^{1-\mu} d x\right\} \\
& =\frac{d}{d s}\left\{0-\frac{1}{1-\mu} \int_{s}^{b} \frac{d v(x)}{d x}(x-s)^{1-\mu} d x\right\} \\
& =\int_{s}^{b} \frac{\frac{d v(x)}{d x} d x}{(x-s)^{\mu}}
\end{aligned}
$$

Now, by substituting $\overline{(C .3)}$ into $[$ C.1], we obtain

$$
\begin{aligned}
\left({ }_{a} \mathcal{D}_{x}^{2 \mu} u, v\right) & =\int_{a}^{b} \widetilde{u}(s)\left\{\frac{1}{\Gamma(1-\mu)}\left(\frac{-d}{d s}\right) \int_{s}^{b} \frac{v(x) d x}{(x-s)^{\mu}}\right\} d s, \\
& =\left(\widetilde{u},{ }_{x} \mathcal{D}_{b}^{\mu} v\right),
\end{aligned}
$$

when ${ }_{x} \mathcal{D}_{b}^{\mu} v$ is well-defined and is integrable in the interval $[a, b]$. $\square$

\section{Appendix D. Proof of Theorem 3.1).}

Proof.

\section{Part A:}

The Jacobi polynomials, $P_{n}^{\alpha, \beta}(\xi)$, can be constructed via the three-term recursion relation. By letting $\beta=-\alpha$, the corresponding tree term recursion reduces to

$$
P_{n+1}^{\alpha,-\alpha}(\xi)=\frac{(2 n+1)}{(n+1)} \xi P_{n}^{\alpha,-\alpha}(\xi)-\frac{\left(n-\alpha^{2}\right)}{n(n+1)} \xi P_{n-1}^{\alpha,-\alpha}(\xi),
$$


and therefore, the Jacobi polynomials evaluated at $\xi=\xi_{0} \in[-1,1]$ are obtained in the following standard form

(D.2)

$$
\begin{array}{ll}
P_{0}^{\alpha,-\alpha}\left(\xi_{0}\right)=\mathbb{P}_{0}(\alpha)=1, & : \text { zeroth order in } \alpha \\
P_{1}^{\alpha,-\alpha}\left(\xi_{0}\right)=\mathbb{P}_{1}(\alpha)=\alpha+\xi_{0}, & : \text { linear in } \alpha \\
P_{2}^{\alpha,-\alpha}\left(\xi_{0}\right)=\mathbb{P}_{2}(\alpha)=\frac{1}{2} \alpha^{2}+\frac{3}{2} \xi_{0} \alpha+\frac{3 \xi_{0}^{2}-1}{2}, & : \text { quadratic in } \alpha \\
P_{3}^{\alpha,-\alpha}\left(\xi_{0}\right)=\mathbb{P}_{3}(\alpha)=\frac{1}{6} \alpha^{3}+\xi_{0} \alpha^{2}+\frac{15 \xi_{0}^{2}-4}{6} \alpha+\frac{5 \xi_{0}^{3}-3 \xi_{0}}{2} . & : \text { cubic in } \alpha
\end{array}
$$

Now, let $n=k$, thus, $P_{k}^{\alpha,-\alpha}\left(\xi_{0}\right)$ and $P_{k-1}^{\alpha,-\alpha}\left(\xi_{0}\right)$ are respectively polynomials of order $k$ and $k-1$ in $\alpha$. Using (D.1) for $n=k+1$, we get

$$
P_{k+1}^{\alpha,-\alpha}\left(\xi_{0}\right)=\frac{(2 k+1)}{(k+1)} \xi_{0} P_{k}^{\alpha,-\alpha}\left(\xi_{0}\right)-\frac{\left(k-\alpha^{2}\right)}{k(k+1)} \xi_{0} P_{k-1}^{\alpha,-\alpha}\left(\xi_{0}\right),
$$

which is a polynomials of order $k+1$ in $\alpha$ due to the second term. Hence, by mathematical induction, $P_{n}^{\alpha,-\alpha}\left(\xi_{0}\right)=\mathbb{P}_{n}(\alpha)$ is a polynomial of order $n$ in $\alpha \forall \xi_{0} \in[-1,1]$. Similarly with the same argument, we can show that $P_{n}^{-\alpha, \alpha}\left(\xi_{0}\right)=\mathbb{P}_{n}(-\alpha)$ is also a polynomial of order $n$ in $\alpha \forall \xi_{0} \in[-1,1]$.

\section{Part B:}

The inner integral of the discrete distributed bilinear form (3.17) can be written as

$$
\begin{aligned}
& \int_{\alpha_{\min }}^{\alpha_{\max }} \phi(\alpha)\left(\frac{2}{T}\right)^{\alpha} \frac{\Gamma(n+\mu)}{\Gamma(n+\eta)} \frac{\Gamma(k+\mu)}{\Gamma(k+\eta)}{ }^{(1)} \mathcal{P}_{n}^{\eta}(\xi){ }^{(2)} \mathcal{P}_{k}^{\eta}(\xi) d \alpha= \\
& \Gamma(n+\mu) \Gamma(k+\mu) \int_{\alpha_{\min }}^{\alpha_{\max }} \phi(\alpha)\left(\frac{2}{T}\right)^{\alpha} \frac{(1+\xi)^{\eta}}{\Gamma(n+\eta)} \frac{(1-\xi)^{\eta}}{\Gamma(k+\eta)} P_{n-1}^{-\eta, \eta}(\xi) P_{k-1}^{\eta,-\eta}(\xi) d \alpha,
\end{aligned}
$$

in which $\eta=\mu-\alpha / 2$. By theorem (3.1) part A, $P_{n-1}^{\frac{\alpha}{2}-\mu, \mu-\frac{\alpha}{2}}(\xi)$ and $P_{k-1}^{\mu-\frac{\alpha}{2}, \frac{\alpha}{2}-\mu}(\xi)$ are polynomials in $\alpha$ of order $n-1$ and $k-1$, respectively, $\forall \xi \in[-1,1]$, and $\mu$ fixed. Thus,

$$
\begin{aligned}
P_{n-1}^{\frac{\alpha}{2}-\mu, \mu-\frac{\alpha}{2}}(\xi) & =\sum_{r=0}^{n-1} \sigma_{r} P_{r}(\alpha), \\
P_{k-1}^{\mu-\frac{\alpha}{2}, \frac{\alpha}{2}-\mu}(\xi) & =\sum_{l=0}^{k-1} \widetilde{\sigma}_{l} P_{l}(\alpha) .
\end{aligned}
$$

By plugging (D.4) and (D.5) into (D.3), we obtain

$$
\begin{aligned}
& \int_{\alpha_{\min }}^{\alpha_{\max }} \phi(\alpha)\left(\frac{2}{T}\right)^{\alpha} \frac{(1+\xi)^{\eta}}{\Gamma(n+\eta)} \frac{(1-\xi)^{\eta}}{\Gamma(k+\eta)} P_{n-1}^{-\eta, \eta}(\xi) P_{k-1}^{\eta,-\eta}(\xi) d \alpha= \\
& \int_{\alpha_{\min }}^{\alpha_{\max }} \phi(\alpha) \mathcal{W}_{k n}^{\xi, \mu}(\alpha) \sum_{r=0}^{n-1} \sigma_{r} P_{r}(\alpha) \sum_{l=0}^{k-1} \widetilde{\sigma}_{l} P_{l}(\alpha) d \alpha,
\end{aligned}
$$

in which

$$
\mathcal{W}_{k n}^{\xi, \mu}(\alpha)=\left(\frac{2}{T}\right)^{\alpha} \frac{(1-\xi)^{\mu-\alpha}}{\Gamma(n+\mu-\alpha)} \frac{(1+\xi)^{\mu-\alpha}}{\Gamma(k+\mu-\alpha)}
$$


is smooth in any compact support in $\left[\alpha_{\min }, \alpha_{\max }\right]$ and its polynomial expansion $\left.\mathcal{W}_{k n}^{\xi, \mu}\right|_{N}(\alpha)=$ $\mathcal{W}_{N}(\alpha)=\sum_{q=0}^{N} \rho_{q} P_{q}(\alpha)$ converges exponentially i.e.,

$$
\left\|\mathcal{W}_{k n}^{\xi, \mu}(\alpha)-\mathcal{W}_{N}(\alpha)\right\| \leq c_{1} \exp \left(-c_{2} N^{c_{3}}\right),
$$

in which $\|$.$\| denotes the L^{2}$-norm in $\left[\alpha_{\min }, \alpha_{\max }\right]$. If the distribution function $\phi \in H^{r}\left(\left[\alpha_{\min }, \alpha_{\max }\right]\right)$, $r>0$, we have the following projection error:

$$
\left\|\phi(\alpha)-\phi_{N}(\alpha)\right\| \leq c_{4} N^{-r}\|\phi\|_{H^{r}\left(\left[\alpha_{\min }, \alpha_{\max }\right]\right)},
$$

where $\phi_{N}(\alpha)=\sum_{n=0}^{N} \widetilde{\rho}_{n} P_{n}(\alpha)$. Consequently, the integrand in (D.6) can be well-approximated via

(D.9)

$$
\phi(\alpha) \mathcal{W}_{k n}^{\xi, \mu}(\alpha) \sum_{r=0}^{n-1} \sigma_{r} P_{r}(\alpha) \sum_{l=0}^{k-1} \widetilde{\sigma}_{l} P_{l}(\alpha) \approx \phi_{N}(\alpha) \mathcal{W}_{N}(\alpha) \sum_{r=0}^{n-1} \sigma_{r} P_{r}(\alpha) \sum_{l=0}^{k-1} \widetilde{\sigma}_{l} P_{l}(\alpha) .
$$

Next, let

$$
\begin{gathered}
\mathcal{I}=\int_{\alpha_{\min }}^{\alpha_{\max }} \phi(\alpha) \mathcal{W}_{k n}^{\xi, \mu}(\alpha) \sum_{r=0}^{n-1} \sigma_{r} P_{r}(\alpha) \sum_{l=0}^{k-1} \widetilde{\sigma}_{l} P_{l}(\alpha) d \alpha, \\
\mathcal{I}_{N}=\int_{\alpha_{\min }}^{\alpha_{\max }} \phi_{N}(\alpha) \mathcal{W}_{N}(\alpha) \sum_{r=0}^{n-1} \sigma_{r} P_{r}(\alpha) \sum_{l=0}^{k-1} \widetilde{\sigma}_{l} P_{l}(\alpha) d \alpha,
\end{gathered}
$$

where $\mathcal{I}_{N}$ can be accurately calculated via

$$
\mathcal{I}_{N}=\sum_{q=1}^{Q} \widetilde{w}_{q} \phi_{N}\left(\alpha_{q}\right) \mathcal{W}_{N}\left(\alpha_{q}\right) \sum_{r=0}^{n-1} \sigma_{r} P_{r}\left(\alpha_{q}\right) \sum_{l=0}^{k-1} \widetilde{\sigma}_{l} P_{l}\left(\alpha_{q}\right)
$$

employing a Gauss-Legendre quadrature rule, provided $Q=2 N$. Thus by Cauchy-schwarz inequality,

$$
\left|\mathcal{I}-\mathcal{I}_{N}\right| \leq \sqrt{\alpha_{\min }-\alpha_{\max }}\left\|\phi \mathcal{W}_{k n}^{\xi, \mu} \sum_{r=0}^{n-1} \sigma_{r} P_{r} \sum_{l=0}^{k-1} \widetilde{\sigma}_{l} P_{l}-\phi_{N} \mathcal{W}_{N} \sum_{r=0}^{n-1} \sigma_{r} P_{r} \sum_{l=0}^{k-1} \widetilde{\sigma}_{l} P_{l}\right\|,
$$


in which

(D.13)

$\left\|\left(\phi \mathcal{W}_{k n}^{\xi, \mu} \sum_{r=0}^{n-1} \sigma_{r} P_{r} \sum_{l=0}^{k-1} \widetilde{\sigma}_{l} P_{l}\right)-\left(\phi_{N} \mathcal{W}_{N} \sum_{r=0}^{n-1} \sigma_{r} P_{r} \sum_{l=0}^{k-1} \widetilde{\sigma}_{l} P_{l}\right)\right\|$,

$\leq\left\|\sum_{r=0}^{n-1} \sigma_{r} P_{r} \sum_{l=0}^{k-1} \widetilde{\sigma}_{l} P_{l}\right\|\left\|\phi \mathcal{W}_{k n}^{\xi, \mu}-\phi_{N} \mathcal{W}_{N}\right\|, \quad$ (by Hölder inequality),

$\leq c_{5}\left\|\phi \mathcal{W}_{k n}^{\xi, \mu}-\phi_{N} \mathcal{W}_{N}\right\|$,

$\leq c_{5}\left\|\left[\left(\phi-\phi_{N}\right)+\phi_{N}\right]\left[\left(\mathcal{W}_{k n}^{\xi, \mu}-\mathcal{W}_{N}\right)+\mathcal{W}_{N}\right]-\phi_{N} \mathcal{W}_{N}\right\|$,

$\leq c_{5}\left\|\left(\phi-\phi_{N}\right)\left(\mathcal{W}_{k n}^{\xi, \mu}-\mathcal{W}_{N}\right)+\phi_{N}\left(\mathcal{W}_{k n}^{\xi, \mu}-\mathcal{W}_{N}\right)+\left(\phi-\phi_{N}\right) \mathcal{W}_{N}+\phi_{N} \mathcal{W}_{N}-\phi_{N} \mathcal{W}_{N}\right\|$,

$\leq c_{5}\left(\left\|\left(\phi-\phi_{N}\right)\left(\mathcal{W}_{k n}^{\xi, \mu}-\mathcal{W}_{N}\right)\right\|+\left\|\phi_{N}\left(\mathcal{W}_{k n}^{\xi, \mu}-\mathcal{W}_{N}\right)\right\|+\left\|\left(\phi-\phi_{N}\right) \mathcal{W}_{N}\right\|\right), \quad$ (by triangle inequality),

$\leq c_{5}\left(\left\|\left(\phi-\phi_{N}\right)\right\|\left\|\left(\mathcal{W}_{k n}^{\xi, \mu}-\mathcal{W}_{N}\right)\right\|+\left\|\phi_{N}\right\|\left\|\left(\mathcal{W}_{k n}^{\xi, \mu}-\mathcal{W}_{N}\right)\right\|+\left\|\left(\phi-\phi_{N}\right)\right\|\left\|\mathcal{W}_{N}\right\|\right)$,

$\leq c_{5}\left(c_{4} N^{-r}\|\phi\|_{H^{r}\left(\left[\alpha_{\min }, \alpha_{\max }\right]\right)} \cdot c_{1} \exp \left(-c_{2} N^{c_{3}}\right)+\left\|\phi_{N}\right\| c_{1} \exp \left(-c_{2} N^{c_{3}}\right)+\left\|\boldsymbol{W}_{N}\right\| c_{4} N^{-r}\|\phi\|_{H^{r}\left(\left[\alpha_{\min }, \alpha_{\max }\right]\right)}\right)$,

(by (D.7) and (D.8),

$\leq c_{6} N^{-r}\|\phi\|_{H^{r}\left(\left[\alpha_{\text {min }}, \alpha_{\text {max }}\right]\right)}$.

Hence, by (D.12) and (D.13) we can show

$$
\left|\mathcal{I}-\mathcal{I}_{N}\right| \leq \mathscr{C} N^{-r}\|\phi\|_{H^{r}\left(\left[\alpha_{\min }, \alpha_{\max }\right]\right)},
$$

and therefore, by (D.4), (D.5), (D.10) and (D.11), we obtain

$$
\begin{aligned}
& \mid \int_{\alpha_{\min }}^{\alpha_{\max }} \phi(\alpha)\left(\frac{2}{T}\right)^{\alpha} \frac{\Gamma(n+\mu)}{\Gamma(n+\eta)} \frac{\Gamma(k+\mu)}{\Gamma(k+\eta)}{ }^{(1)} \mathcal{P}_{n}^{\eta}(\xi){ }^{(2)} \mathcal{P}_{k}^{\eta}(\xi) d \alpha \\
& \quad-\sum_{q=1}^{Q} \widetilde{w}_{q} \phi_{N}\left(\alpha_{q}\right)\left(\frac{2}{T}\right)^{\alpha_{q}} \frac{\Gamma(n+\mu)}{\Gamma\left(n+\eta_{q}\right)} \frac{\Gamma(k+\mu)}{\Gamma\left(k+\eta_{q}\right)}{ }^{(1)} \mathcal{P}_{n}^{\eta_{q}}(\xi){ }^{(2)} \mathcal{P}_{k}^{\eta_{q}}(\xi) \mid \\
& \leq \mathscr{C} Q^{-r}\|\phi\|_{H^{r}\left(\left[\alpha_{\min }, \alpha_{\text {max }}\right]\right)} .
\end{aligned}
$$

\section{Part C:}

If $\phi(\alpha)$ is smooth, then the approximation $\phi_{N}(\alpha)$, in (D.8), converges with an exponential accuracy and so does the norm in (D.13). Thus,

$$
\left|I-I_{N}\right| \leq \mathscr{C}_{1} \exp \left(-\mathscr{C}_{2} N^{\mathscr{C}_{3}}\right),
$$

and therefore, the quadrature rule becomes exponentially accurate in $Q$.

प 


\section{REFERENCES}

[1] R. Askey and J. Fitch, Integral representations for Jacobi polynomials and some applications, Journal of Mathematical Analysis and Applications, 26 (1969), pp. 411-437.

[2] T.M. Atanackovic, L. Oparnica, and S. Pilipović, Distributional framework for solving fractional differential equations, Integral Transforms and Special Functions, 20 (2009), pp. 215-222.

[3] T.M. Atanackovic, S. Pilipovic, and D. Zorica, Existence and calculation of the solution to the time distributed order diffusion equation, Physica Scripta, 2009 (2009), p. 014012.

[4] T. M. Atanackovic, S. Pilipovic, and D. Zorica, Time distributed-order diffusion-wave equation. i. Volterratype equation, Proceedings of the Royal Society A: Mathematical, Physical and Engineering Science, 465 (2009), pp. 1869-1891.

[5] B. Baeumer, D. A. Benson, M.M. Meerschaert, and S. W. Wheatcraft, Subordinated advection-dispersion equation for contaminant transport, Water Resources Research, 37 (2001), pp. 1543-1550.

[6] A. H. Bhrawy, E. H. Doha, D. Baleanu, and S. S. Ezz-Eldien, A spectral tau algorithm based on Jacobi operational matrix for numerical solution of time fractional diffusion-wave equations, Journal of Computational Physics, 293 (2015), pp. 142-156.

[7] J. CAO AND C. XU, A high order schema for the numerical solution of the fractional ordinary differential equations, Journal of Computational Physics, 238 (2013), pp. 154-168.

[8] S. Chen, J. Shen, ANd L. WANG, Generalized Jacobi functions and their applications to fractional differential equations, arXiv preprint arXiv:1407.8303, (2014).

[9] D. del Castillo-Negrete, B. A. Carreras, and V. E. Lynch, Fractional diffusion in plasma turbulence, Physics of Plasmas (1994-present), 11 (2004), pp. 3854-3864.

[10] K. Diethelm and N. J. Ford, Numerical solution methods for distributed order differential equations, Fractional Calculus and Applied Analysis, 4 (2001), pp. 531-542.

[11] _ Numerical analysis for distributed-order differential equations, Journal of Computational and Applied Mathematics, 225 (2009), pp. 96-104.

[12] Kai Diethelm and Neville J Ford, Numerical analysis for distributed-order differential equations, Journal of Computational and Applied Mathematics, 225 (2009), pp. 96-104.

[13] K. Diethelm, N. J. Ford, And A. D. Freed, Detailed error analysis for a fractional Adams method, Numerical Algorithms, 36 (2004), pp. 31-52.

[14] P. L. T. Duong, E. KwoK, AND M. LeE, Deterministic analysis of distributed order systems using operational matrix, Applied Mathematical Modelling, 40 (2016), pp. 1929-1940.

[15] A. Ern and J. Guermond, Theory and practice of finite elements, vol. 159, Springer Science \& Business Media, 2013

[16] N. J. Ford, M. L. Morgado, and M. Rebelo, A numerical method for the distributed order time-fractional diffusion equation, in Fractional Differentiation and Its Applications (ICFDA), 2014 International Conference on, IEEE, 2014, pp. 1-6.

[17] _ An implicit finite difference approximation for the solution of the diffusion equation with distributed order in time, Electronic Transactions on Numerical Analysis, 44 (2015), pp. 289-305.

[18] G. GaO, H. Sun, AND Z. Sun, Some high-order difference schemes for the distributed-order differential equations, Journal of Computational Physics, 298 (2015), pp. 337-359.

[19] G. GAO AND Z. Sun, Two alternating direction implicit difference schemes with the extrapolation method for the two-dimensional distributed-order differential equations, Computers \& Mathematics with Applications, 69 (2015), pp. 926-948.

[20] - Two unconditionally stable and convergent difference schemes with the extrapolation method for the one-dimensional distributed-order differential equations, Numerical Methods for Partial Differential Equations, (2015).

[21] R. Gorenflo, F. Mainardi, D. Moretti, and P. Paradisi, Time fractional diffusion: a discrete random walk approach, Nonlinear Dynamics, 29 (2002), pp. 129-143.

[22] I. Hashim, O. Abdulaziz, and S. Momani, Homotopy analysis method for fractional ivps, Communications in Nonlinear Science and Numerical Simulation, 14 (2009), pp. 674-684.

[23] X. Hu, F. Liu, I. Turner, AND V. AnH, An implicit numerical method of a new time distributed-order and two-sided space-fractional advection-dispersion equation, Numerical Algorithms, (2015), pp. 1-15.

[24] J Huang, Y TANG, AND L VAzQuez, Convergence analysis of a block-by-block method for fractional differential equations, Numer. Math. Theor. Methods Appl., 5 (2012), pp. 229-241.

[25] M. Inc, The approximate and exact solutions of the space-and time-fractional Burgers equations with initial conditions by variational iteration method, Journal of Mathematical Analysis and Applications, 345 (2008), pp. 476-484.

[26] H. Jafari and V. Daftardar-GejJi, Solving linear and nonlinear fractional diffusion and wave equations by Adomian decomposition, Applied Mathematics and Computation, 180 (2006), pp. 488-497.

[27] A. Jaishankar and G. H. McKinley, Power-law rheology in the bulk and at the interface: quasi-properties and fractional constitutive equations, Proceedings of the Royal Society A: Mathematical, Physical and 
Engineering Science, 469 (2013), p. 20120284.

[28] Aditya Jaishankar and Gareth H McKinley, A fractional $k$-bkz constitutive formulation for describing the nonlinear rheology of multiscale complex fluids, Journal of Rheology (1978-present), 58 (2014), pp. 1751-1788.

[29] R. Jha, P. K. Kaw, D. R. Kulkarni, J. C. Parikh, and ADitya Team, Evidence of lévy stable process in tokamak edge turbulence, Physics of Plasmas (1994-present), 10 (2003), pp. 699-704.

[30] M. M. KHADER, On the numerical solutions for the fractional diffusion equation, Communications in Nonlinear Science and Numerical Simulation, 16 (2011), pp. 2535-2542.

[31] M. M. Khader AND A. S. Hendy, The approximate and exact solutions of the fractional-order delay differential equations using Legendre pseudospectral method, International Journal of Pure and Applied Mathematics, 74 (2012), pp. 287-297.

[32] A. A. Kilbass, H. M. SRivastava, and J. J. Trujillo, Theory and Applications of Fractional Differential Equations, Amsterdam, Netherlands:Elsevier, 2006.

[33] TAM Langlands and BI HeNRY, The accuracy and stability of an implicit solution method for the fractional diffusion equation, Journal of Computational Physics, 205 (2005), pp. 719-736.

[34] X. Li and B. Wu, A numerical method for solving distributed order diffusion equations, Applied Mathematics Letters, 53 (2016), pp. 92-99.

[35] X. LI AND C. XU, A space-time spectral method for the time fractional diffusion equation, SIAM Journal on Numerical Analysis, 47 (2009), pp. 2108-2131.

[36] - Existence and uniqueness of the weak solution of the space-time fractional diffusion equation and a spectral method approximation, Communications in Computational Physics, 8 (2010), p. 1016.

[37] Y. LIN AND C. Xu, Finite difference/spectral approximations for the time-fractional diffusion equation, Journal of Computational Physics, 225 (2007), pp. 1533-1552.

[38] C. LuBich, On the stability of linear multistep methods for volterra convolution equations, IMA Journal of Numerical Analysis, 3 (1983), pp. 439-465.

[39] Сн Lubich, Discretized fractional calculus, SIAM Journal on Mathematical Analysis, 17 (1986), pp. 704-719.

[40] F. Mainardi, G. Pagnini, and R. Gorenflo, Some aspects of fractional diffusion equations of single and distributed order, Applied Mathematics and Computation, 187 (2007), pp. 295-305.

[41] F. C. Meral, T. J. Royston, and R. Magin, Fractional calculus in viscoelasticity: an experimental study, Communications in Nonlinear Science and Numerical Simulation, 15 (2010), pp. 939-945.

[42] R. Metzler AND J. Klafter, The random walk's guide to anomalous diffusion: a fractional dynamics approach, Physics Reports, 339 (2000), pp. 1-77.

[43] K. S. Mrlere and B. Ross, An Introduction to the Fractional Calculus and Fractional Differential Equations, New York, NY:John Wiley and Sons, Inc., 1993.

[44] M. Morgado and M. Rebelo, Numerical approximation of distributed order reaction-diffusion equations, Journal of Computational and Applied Mathematics, 275 (2015), pp. 216-227.

[45] M. NAGHIBOLHosseinI, Estimation of outer-middle ear transmission using DPOAEs and fractional-order modeling of human middle ear, PhD thesis, City University of New York, NY., 2015.

[46] I PoduubNy, Fractional Differential Equations, San Diego, CA, USA: Academic Press, 1999.

[47] E. A. RAWASHDEH, Numerical solution of fractional integro-differential equations by collocation method, Applied Mathematics and Computation, 176 (2006), pp. 1-6.

[48] S. G. Samko, A. A. Kilbas, and O. I. Marichev, Fractional Integrals and Derivatives: Theory and Applications, Gordon and Breach, Yverdon, 1993.

[49] J. M. SANZ-SERNA, A numerical method for a partial integro-differential equation, SIAM Journal on Numerical Analysis, 25 (1988), pp. 319-327.

[50] K. R. Sreenivasan and R. A. Antonia, The phenomenology of small-scale turbulence, Annual Review of Fluid Mechanics, 29 (1997), pp. 435-472.

[51] T. SRokowsкi, Lévy flights in nonhomogeneous media: distributed-order fractional equation approach, Physical Review E, 78 (2008), p. 031135.

[52] N. Sugimoto, Burgers equation with a fractional derivative; hereditary effects on nonlinear acoustic waves, Journal of Fluid Mechanics, 225 (1991), p. 4.

[53] Z. Sun AND X. Wu, A fully discrete difference scheme for a diffusion-wave system, Applied Numerical Mathematics, 56 (2006), pp. 193-209.

[54] N. H. Sweilam, M. M. Khader, ANd R. F. Al-Bar, Numerical studies for a multi-order fractional differential equation, Physics Letters A, 371 (2007), pp. 26-33.

[55] H. Wang, K. Wang, and T. Sircar, A direct o $($ nlog $2 n)$ finite difference method for fractional diffusion equations, Journal of Computational Physics, 229 (2010), pp. 8095-8104.

[56] H. WANG AND X. ZHANG, A high-accuracy preserving spectral galerkin method for the dirichlet boundary-value problem of variable-coefficient conservative fractional diffusion equations, Journal of Computational Physics, 281 (2015), pp. 67-81.

[57] K. WANG AND H. WANG, A fast characteristic finite difference method for fractional advection-diffusion equations, Advances in Water Resources, 34 (2011), pp. 810-816. 
[58] H. YE, F. Liu, AND V. ANH, Compact difference scheme for distributed-order time-fractional diffusion-wave equation on bounded domains, Journal of Computational Physics, 298 (2015), pp. 652-660.

[59] M. Zayernouri, M. Ainsworth, and G. E. Karniadakis, Tempered fractional sturm-liouville eigenproblems, SIAM Journal on Scientific Computing, 37 (2015), pp. A1777-A1800.

[60] M. Zayernouri, W. Cao, Z. Zhang, and G. E. Karniadakis, Spectral and discontinuous spectral element methods for fractional delay equations, SIAM Journal on Scientific Computing, 36 (2014), pp. B904B929.

[61] M. ZaYernouri and G. E. Karniadakis, Fractional Sturm-Liouville eigen-problems: theory and numerical approximations, J. Comp. Physics, 47-3 (2013), pp. 2108-2131.

[62] _ Discontinuous spectral element methods for time-and space-fractional advection equations, SIAM Journal on Scientific Computing, 36 (2014), pp. B684-B707.

[63] _ Exponentially accurate spectral and spectral element methods for fractional odes, J. Comp. Physics, 257 (2014), pp. 460-480.

[64] _ - Fractional spectral collocation method, SIAM Journal on Scientific Computing, 36 (2014), pp. A40A62.

[65] _ Fractional spectral collocation methods for linear and nonlinear variable order fpdes, Journal of Computational Physics, 293 (2015), pp. 312-338.

[66] M. Zayernouri and A. Matzavinos, Fractional Adams-Bashforth/Moulton methods: An application to the fractional Keller-Segel chemotaxis system, Journal of Computational Physics-In Press, (2016).

[67] F. Zeng, C. Li, F. LiU, AND I. Turner, Numerical algorithms for time-fractional subdiffusion equation with second-order accuracy, SIAM Journal on Scientific Computing, 37 (2015), pp. A55-A78.

[68] H. Zhang, F. Liu, and V. ANH, Galerkin finite element approximation of symmetric space-fractional partial differential equations, Applied Mathematics and Computation, 217 (2010), pp. 2534-2545. 